$\checkmark$ Research Square

\title{
Different hotspot p53 mutants exert distinct phenotypes and predict outcome of colorectal cancer patients
}

Ori Hassin

Weizmann Institute of Science

Nishanth Belugali Nataraj

Weizmann Institute of Science https:// orcid.org/0000-0003-2970-6739

Michal Shreberk-Shaked

Weizmann Institute of Science

Yael Aylon

Weizmann Institute of Science https://orcid.org/0000-0001-5821-9548

\section{Rona Yaeger}

Memorial Sloan Kettering Cancer Center

Giulia Fontemaggi

IRCCS Regina Elena National Cancer Institute

\section{Saptaparna Mukherjee}

Weizmann Institute of Science

\section{Martino Maddalena}

Weizmann Institute of Science

\section{Giuseppe Mallel}

Curesponse Ltd https://orcid.org/0000-0002-8673-7526

\section{Anat Gershon}

Weizmann Institute of Science

Inna Grosheva

Weizmann Institute of Science

\section{Ester Feldmesser}

Weizmann Institute of Science https://orcid.org/0000-0002-7017-4861

\section{Ofra Golani}

Weizmann Institute of Science https://orcid.org/0000-0002-9793-236X

\section{Giovanni Blandino}

IRCCS Regina Elena National Cancer Institute

\section{David Kelsen}

Memorial Sloan Kettering Cancer Center https://orcid.org/0000-0001-6139-438X

\section{Yosef Yarden}


Weizmann Institute of Science https://orcid.org/0000-0003-4168-7884

\section{Moshe Oren ( $\nabla$ moshe.oren@weizmann.ac.il )}

Weizmann Institute of Science

Article

Keywords: colorectal cancer (CRC), TP53, genetics, mutation

Posted Date: June 4th, 2021

DOI: https://doi.org/10.21203/rs.3.rs-523301/v1

License: (1) This work is licensed under a Creative Commons Attribution 4.0 International License. Read Full License

Version of Record: A version of this preprint was published at Nature Communications on May 19th, 2022. See the published version at https://doi.org/10.1038/s41467-022-30481-7. 


\section{Abstract}

Colorectal cancer (CRC) is the third most common cancer worldwide. The TP53 gene is mutated in approximately $60 \%$ of all CRC cases. Sporadic CRC is characterized by high prevalence of TP53 hotspot missense mutations. In particular, over 20 percent of all TP53-mutated CRC tumors carry either the $p 53^{\mathrm{R} 175 \mathrm{H}}$ structural mutant or the $\mathrm{p} 53^{\mathrm{R} 273 \mathrm{H}}$ DNA contact mutant. Importantly, clinical data analysis suggests that CRC tumors harboring p53 R273 mutations are more prone to progress to metastatic disease than those with R175 mutations, in association with decreased survival. By combining in vitro CRC cell line models and human CRC data mining, we identified a unique transcriptional signature orchestrated by $\mathrm{p} 53^{\mathrm{R} 273 \mathrm{H}}$, implicating activation of oncogenic signaling pathways and predicting worse patient outcome. Concordantly, $\mathrm{p} 53^{\mathrm{R} 273 \mathrm{H}}$ selectively promotes rapid CRC cell spreading, migration and invasion in vitro and metastasis in vivo. Mechanistically, the transcriptional output of $p 53^{\mathrm{R} 273 \mathrm{H}}$ is associated with, and presumably driven by, its preferential binding to regulatory elements of R273 signature genes. Together, this demonstrates that different TP53 missense mutations contribute differently to cancer progression, and that $\mathrm{p} 53^{\mathrm{R} 273 \mathrm{H}}$ possesses distinct gain-of-function activities in CRC that bear on disease course and possibly on patient management strategy. Given that practically all current analytical cancer gene panels include TP53, elucidation of the differential impact of distinct TP53 mutations on disease features is expected to make information on TP53 mutations more actionable and holds potential for better precision-based medicine.

\section{Introduction}

The TP53 gene, encoding the p53 tumor suppressor protein, is frequently mutated in many types of human cancer $(1,2)$. The most common type of TP53 mutations are missense mutations, leading to a single amino acid substitution in an otherwise intact p53 protein. In addition, TP53 nonsense and frameshift mutations, usually resulting in production of truncated p53 proteins, are also fairly common in cancer (3). The common and arguably most important consequence of all these different types of mutations is the partial or complete loss of the tumor suppressor effects of the wild type (wt) p53 protein. Yet, there is growing evidence that missense TP53 mutations may often also confer upon the mutant p53 (mutp53) proteins oncogenic gain-of-function (GOF) properties, which can actively contribute to cancerrelated processes $(4,5,6,7,8)$.

The spectrum of TP53 missense mutations in human cancer comprises hundreds of different variants, although a small number of hotspot mutations are observed more frequently (9). Broadly speaking, cancer-associated p53 missense mutant proteins can be divided into two main classes: (A) structural mutants, where the mutation causes misfolding of the protein and leads to a significant conformational alterations within p53's DNA binding domain (DBD), and (B) DNA contact mutants, where the overall structure of the DBD is only minimally perturbed, but the mutant protein loses its ability to engage in highaffinity sequence-specific interactions with p53 binding sites within the DNA (10). Both mutp53 classes 
fail to activate canonical wtp53 target genes, but can modify the cell transcriptome through proteinprotein interactions that involve a multitude of transcription factors and other DNA binding proteins $(5,7)$.

While most of the studies on mutp53 have addressed features shared by all common mutants, there also is evidence for mutant-specific effects $(5,11,12)$. Notably, knock-in mice harboring different p53 mutations exhibit non-identical tumor phenotypes: $\mathrm{p} 53^{\mathrm{R} 270 \mathrm{H} /+}$ mice, corresponding to the human p53 ${ }^{\mathrm{R} 273 \mathrm{H}}$ DNA contact hotspot mutation, show increased incidence of carcinomas and $\mathrm{B}$ cell lymphomas compared to $\mathrm{p} 53+/-$ mice, while $\mathrm{p} 53^{\mathrm{R} 172 \mathrm{H} /+}$ mice, corresponding to the human $\mathrm{p} 53^{\mathrm{R} 175 \mathrm{H}}$ structural hotspot mutation, develop mainly osteosarcomas (13). However, the clinical implications of such mutantspecific differences remain largely unknown.

Colorectal cancer (CRC) is the 2 nd most common cause of cancer-related deaths worldwide (14). The malignant progression of CRC is driven largely by the sequential accumulation of genetic alterations, affecting both oncogenes and tumor suppressor genes (15). Like other cancer types, CRC displays a wide spectrum of TP53 mutations, which are observed in approximately $60 \%$ of all CRC tumors and are usually associated with the transition from large adenoma to invasive carcinoma (15).

In the present study, we set out to compare the impact of the two most common hotspot TP53 mutations in $\mathrm{CRC}, \mathrm{p} 53^{\mathrm{R} 273 \mathrm{H}}$ and $\mathrm{p} 53^{\mathrm{R} 175 \mathrm{H}}$. Interestingly, we found marked differences between the effects of these two mutants. Specifically, $\mathrm{p} 53^{\mathrm{R} 273 \mathrm{H}}$ but not $\mathrm{p} 53^{\mathrm{R} 175 \mathrm{H}}$ can orchestrate a unique transcriptional program, which drives oncogenic signaling pathways, leads to more aggressive disease, and is associated with significant differences in patient survival. Better understanding of the distinct contributions of different TP53 mutants might guide better CRC patient management and treatment decisions.

\section{Results}

\section{p53 R273 mutants are associated with more aggressive colorectal tumors relative to R175 mutants}

Compared to most other cancers, in colorectal cancer (CRC) the relative representation of "hotspot" missense mutations among carriers of TP53 mutations is particularly high. Specifically, missense mutations in the four most commonly mutated p53 residues (R175, R248, R273 and R282) comprise approximately $37 \%$ of all TP53 mutations in this type of cancer (Fig. S1a). In contrast, mutations in these four residues encompass only $17 \%$ of all TP53 mutations in all other cancer types together. Although this might be simply due to the mutational signature of particular carcinogens, it might also suggest a more significant GOF effect of such missense mutations in CRC.

One obvious question is whether different hotspot mutations may exert different effects on disease features and patient outcome. To address this question, we set out to compare R175 structural mutations to R273 DNA contact mutations. Notably, these mutations together represent over $20 \%$ of all CRC tumors harboring TP53 mutations, as compared to only approximately $10 \%$ in all other cancers (Fig $1 \mathrm{~A}$ ). We analyzed clinical data from several patient cohorts, using the TCGA and ICGC open-source platforms as 
well as additional published datasets $(16,17,18)$ (Supplementary Table 1). Remarkably, while R175 mutations are significantly more frequent than R273 mutations in early disease stages, the predominance of R175 mutations is abolished at later stages (Fig. 1b). This suggests that, relative to R175 mutations, R273 mutations might accelerate disease progression from early stages to advanced stages, involving cancer cell spreading to nearby lymph nodes (stage 3) and metastases to distant organs (stage 4).

Interestingly, when we analyzed the MSKCC CRC dataset, comprising 1134 cases of which $90 \%$ were metastatic (19), we found that while both R175 and R273 mutants exhibited a similar percentage of liver, lung and lymph node first site metastases (data not shown), R273 mutants were significantly more associated with tumors that metastasize first to less common sites such as brain, bone, pelvis, peritoneum and gynecological sites (Fig. 1C). Importantly, unlike liver and lung metastases, metastatic lesions in these sites are usually considered unresectable, and thus incurable. Indeed, many studies have linked the presence of metastases at those sites to worse survival $(20,21,22)$. Furthermore, R273 mutants were found to be significantly associated with multiple metastatic sites at the time of diagnosis of metastatic disease (Fig. 1d), further supporting the notion that R273 mutants selectively augment the metastatic capacity of CRC cancer cells. Importantly, R273 mutants were associated with significantly shorter disease-specific overall survival than R175 mutants (Fig 1e), regardless of patient age, sex, tumor location or presence of KRAS mutations (Fig $1 \mathrm{f}$ and Supplementary Table 2). Interestingly, while the impact of R273 mutations on overall survival was prominent in CRC patients presenting at stages 1-3 (Fig. S1b), it was not seen anymore when the patients presented with stage 4 disease (Fig. S1c); this is consistent with the notion that the main effect of R273 mutations is on the rate of progression from early stage CRC to advanced disease .

To explore the possibility that R273 mutant tumors might be associated with a particular mutational landscape, which may account for the observed clinical effects, we compared the co-occurrence of the most common gene mutations in CRC with either R175 or R273 mutations. Notably, other than SMAD4 mutations which showed a mild co-occurrence with R273 mutations $(P=0.02)$, all other gene mutations were not differentially enriched in R273 mutated vs R175 mutated tumors (Fig S1d).

In sum, compared to R175 mutations, R273 mutations are preferentially associated with more advanced disease, higher rate of multiple and uncommon metastases, and shorter patient survival.

\section{$\underline{\mathrm{p} 53^{\mathrm{R} 273 \mathrm{H}}}$ orchestrates a distinct transcriptional signature}

We next wished to elucidate the molecular mechanisms underpinning the differential impact of R273 vs R175 mutants in CRC, and to assess whether R273 mutations confer a true GOF. To that end, we utilized CRC-derived SW480 cells. SW480 is a microsatellite stable cell line, harboring APC and KRAS mutations; hence, it properly represents sporadic CRC. SW480 cells endogenously express two p53 mutants: p53 ${ }^{\mathrm{R} 273 \mathrm{H}}$, and the less common p53 ${ }^{\mathrm{P} 309 \mathrm{~S}}$ (23). SW480 cells depleted of their endogenous mutp53 by CRISPR/Cas9-mediated knockout (p53KO) were stably transduced with either $\mathrm{p} 53^{\mathrm{R} 273 \mathrm{H}}$ or $\mathrm{p} 53^{\mathrm{R} 175 \mathrm{H}}$ (Fig. 2a). Western blot analysis confirmed comparable overexpression of both mutants (Fig. 2b). As mutp53 
GOF often involves changes in the cell transcriptome, we next subjected the different SW480 cell pools to RNA sequencing (RNA-seq) analysis, using the MARS-seq protocol (24). Clustering analysis revealed substantial differences between the transcriptome of the R273H cells and the parental p53KO cells (Fig. 2c). Surprisingly, overexpression of $\mathrm{p} 53^{\mathrm{R} 175 \mathrm{H}}$ had rather limited impact on the transcriptome of these cells (Fig. 2c). By comparing the observed transcriptional profiles, we generated a gene signature comprising 140 genes upregulated by $p 53^{\mathrm{R} 273 \mathrm{H}}$ relative to both $\mathrm{p} 53^{\mathrm{R} 175 \mathrm{H}}$ and $\mathrm{p} 53 \mathrm{KO}$ cells. This gene signature was defined as the "R273 signature" (Fig. 2d).

To further validate our conclusions, we adopted an alternative approach wherein SW480 cells were stably transduced with shRNA directed against the 3' UTR of the TP53 gene (shp53), followed by stable overexpression of shRNA-resistant $\mathrm{p} 53^{\mathrm{R} 175 \mathrm{H}}$ or $\mathrm{p} 53^{\mathrm{R} 273 \mathrm{H}}$ (Fig. 2e). The resultant cell pools were subjected to MARS-seq analysis as above. Clustering analysis of the data confirmed that, also by this approach, $\mathrm{p} 53^{\mathrm{R} 273 \mathrm{H}}$ had a stronger effect on the SW480 cell transcriptome than $\mathrm{p} 53^{\mathrm{R} 175 \mathrm{H}}$ (Fig. S2a). Importantly, the "R273 signature", deduced from the reconstituted p53KO cells, was strongly correlated with the differences in gene expression between the R273H-reconstituted shp53 cells and the control (Fig. 2f) or R175H-reconstituted (Fig. 2g) cells, as determined by gene set enrichment analysis (GSEA).

Lastly, since the above RNA-seq analyses were done with ectopically overexpressed p53 mutants, we quantified the relative expression of representative R273 signature genes by RT-qPCR analysis in control (expressing endogenous mutp53) and p53KO SW480 cells (Western blot in Fig. S2b). As seen in Fig S2c, all tested genes were significantly downregulated in the knockout cells, consistent with their being positively regulated by $\mathrm{p} 53^{\mathrm{R} 273 \mathrm{H}}$. Moreover, comparison by GSEA of our R273 signature to published RNAseq data of SW480 cells before and after shRNA-mediated p53 knockdown (25) confirmed significantly higher expression of the R273 signature in the control cells, which harbor endogenous $\mathrm{p} 53^{\mathrm{R} 273 \mathrm{H}}$ (Fig. S2d). Thus, $\mathrm{p} 53^{\mathrm{R} 273 \mathrm{H}}$ drives a distinct transcriptional program in SW480 cells.

\section{The R273 signature is upregulated in multiple CRC cell lines and tumors and is associated with poor survival}

To assess the generality of the R273 signature we interrogated experimentally three additional CRCderived cell lines, by expressing $\mathrm{p} 53^{\mathrm{R} 273 \mathrm{H}}$ and $\mathrm{p} 53^{\mathrm{R} 175 \mathrm{H}}$ ectopically in HCT116 and RKO cells depleted of their endogenous wtp53 (KO), and COLO-205 cells endogenously expressing truncated p53 (Fig. $3 \mathrm{a}$ and Fig. S3a,c). Reassuringly, RT-qPCR analysis of representative R273 signature genes confirmed that, in all three cell lines, $\mathrm{p} 53^{\mathrm{R} 273 \mathrm{H}}$ selectively upregulated these genes, albeit to varying extents (Fig. 3b, Fig. $\mathrm{S} 3 \mathrm{~b}, \mathrm{~d})$. Moreover, using the cancer cell line encyclopedia (CCLE) database, we found that the R273 signature is significantly upregulated in CRC cell lines harboring R273 mutations, compared to CRC lines carrying protein-truncating TP53 mutations (Fig. 3c). The CCLE includes only three R175-mutated CRC lines; while their analysis indicated a similar trend as above, statistical significance could not be reached $(p=0.067$; data not shown). 
We next wished to extend these findings to human CRC tumors. Importantly, GSEA analysis of the TCGA CRC cohort revealed that tumors harboring R273 mutations displayed significantly higher expression of the R273 signature than those with R175 mutations (Fig. 3d). Comparison of the R273-mutated tumors to tumors carrying truncating TP53 mutations yielded a similar trend, but the difference did not reach statistical significance (data not shown). Of note, the truncating mutations group is very heterogeneous, and not all cases may resemble a p53-null state. Yet, tumors with extremely low p53 mRNA levels, presumably owing to nonsense-mediated decay (3), are more likely to approximate true nulls. Indeed, when we included only truncating mutation cases displaying greatly reduced steady-state p53 mRNA, unequivocal association of R273-mutated tumors with the R273 signature was clearly evident (Fig. 3e). Interestingly, analysis of the entire set of CRC tumors revealed a remarkable degree of positive correlations between the expression levels of the genes comprising the R273 signature, which was not observed in three independent control signatures (Fig S3e,f). This suggests that many of the genes comprising the R273 signature may be subject to common transcriptional or post-transcriptional regulatory mechanisms.

Guinney et al. have recently employed comprehensive data analysis to define four consensus molecular subtypes (CMS) for colorectal cancer (26). Remarkably, when we compared our R273 signature with the cell-intrinsic transcriptional signatures of the four CMS subtypes, as determined by Sveen et al. (27), the R273 signature displayed a strong $(R=0.66)$ and significant $(p<2.2 \mathrm{e}-16)$ correlation with the CMS4 signature (Fig S4a). Furthermore, GSEA analysis confirmed that CRC tumors harboring R273 mutations are significantly associated with the CMS4 gene signature compared to tumors harboring R175 mutations or truncating mutation (Fig S4b). Interestingly, the GSEA analysis revealed that tumors harboring R175 mutations are significantly associated with the CMS2 gene signature, when compared to tumors harboring either R273 or truncating mutations (Fig S4c). Hence, R273 mutations and R175 mutations are differentially associated with distinct CRC molecular subtypes, implicating markedly different cancer-promoting biological processes (26).

Importantly, comparison of TCGA CRC tumors displaying high (upper quartile) R273 signature vs those with low (bottom quartile) signature revealed that high R273 signature was significantly associated with late-stage disease (Fig. 3f) and shorter patient survival (Fig. 3g). Furthermore, multivariate Cox regression analysis for overall survival, including age, sex, tumor location and the presence of KRAS mutations, demonstrated that high expression of the R273 signature is an independent prognostic factor (multivariate hazard ratio 2.314; 95\% confidence interval 1.344-3.977; P=0.002; Supplementary Table 3).

In sum, the R273 gene signature is broadly enriched in CRC cells and tumors harboring R273 mutations, and is correlated with shorter patient survival. This further supports the hypothesis that the transcriptional output directed by R273 mutants endows CRC tumors with more aggressive features, which adversely affect patient outcome.

\section{R273 mutants selectively_promote cell spreading, migration and invasion}


To elucidate oncogenic pathways that may contribute to the clinical impact of R273 mutations, we subjected the R273 signature to Gene Ontology analysis by METASCAPE (28). Interestingly, many observed pathways were directly or indirectly related to cytoskeleton dynamics (Fig. 4a), which is often associated with cancer-related properties such as cell adhesion, spreading, migration and invasion $(29,30,31,32)$. Specifically, the Rho signaling pathway, ranking high in this analysis, can promote cancer by driving actin cytoskeleton remodeling and augmenting cell migration, survival, polarity, and more $(33,34)$.

Phenotypically, the morphology of SW480 cells expressing $p 53^{\mathrm{R} 273 \mathrm{H}}$ differed visibly from that of parental knockout cells or $\mathrm{p} 53^{\mathrm{R} 175 \mathrm{H}}$ expressors. This was evident as accelerated spreading, confirmed by timelapse microscopy (Fig. 4b and Supplementary movies 1-3). Similar observations were made with RKO cells, depleted of their endogenous wtp53 and reconstituted with either $\mathrm{p} 53^{\mathrm{R} 175 \mathrm{H}}$ or $\mathrm{p} 53^{\mathrm{R} 273 \mathrm{H}}$ (Fig S5a). Importantly, RNA-seq analysis six hours after plating (Fig. S5b) showed that already at this early time point the R273 signature was upregulated in the $\mathrm{p} 53^{\mathrm{R} 273 \mathrm{H}}$ expressors to a similar extent as after 24 hours. This supports the notion that the inherent gene expression pattern dictated by $\mathrm{p} 53^{\mathrm{R} 273 \mathrm{H}}$ drives cell spreading, rather than being secondary to it.

Cell cycle analysis did not reveal differences between the effects of $\mathrm{p}^{\mathrm{R}} \mathrm{R}^{\mathrm{2} 23 \mathrm{H}}$ and $\mathrm{p} 53^{\mathrm{R} 175 \mathrm{H}}$ (Fig. S5c). However, the $\mathrm{p}^{\mathrm{R}} 3^{\mathrm{R} 273 \mathrm{H}}$ expressors displayed a significant increase in cell migration (Fig. 4c,d) and invasion (Fig. $4 \mathrm{e}, \mathrm{f}$ ), relative to $\mathrm{p} 53^{\mathrm{R} 175 \mathrm{H}}$ expressors or knockout cells. Moreover, while both $\mathrm{p} 53^{\mathrm{R} 273 \mathrm{H}}$ and $\mathrm{p} 53^{\mathrm{R} 175 \mathrm{H}}$ augmented the migration of $\mathrm{p} 53$-depleted $\mathrm{RKO}$ cells, the effect of $\mathrm{p} 53^{\mathrm{R} 273 \mathrm{H}}$ was significantly greater (Fig S5d,e). Thus, $\mathrm{p5} 3^{\mathrm{R} 273 \mathrm{H}}$ preferentially promotes cell spreading, migration and invasion.

Rho signaling is one of the top enriched pathways in the R273 signature (Fig. 4a). In agreement, a Rho proteins GTPase activation assay confirmed that overexpression $\mathrm{p} 53^{\mathrm{R} 273 \mathrm{H}}$ of in SW480 cells augmented the activation of both $\mathrm{Cdc} 42$ and Rac1, relative to $\mathrm{p} 53^{\mathrm{R} 175 \mathrm{H}}$ overexpressors (Fig. 4g). Interestingly, RhoA activation was not differentially affected. Importantly, the migratory phenotype of $p 53^{R 273 H}$ overexpressors was completely abolished by treatment with the Rac1/Cdc42 inhibitor MBQ-167 (Fig. 4h). Hence, $\mathrm{p} 53^{\mathrm{R} 273 \mathrm{H}}$ selectively drives Rac1/Cdc42-dependent cancer cell migration.

\section{p53 $\underline{\text { R273H }}$ preferentially_promotes metastasis}

We next wished to assess whether the differential impact of $\mathrm{p} 53^{\mathrm{R} 273 \mathrm{H}}$ in vitro is also reflected in a more aggressive phenotype in vivo. To that end, SW480 cells ectopically expressing either $\mathrm{p} 53^{\mathrm{R} 175 \mathrm{H}}$ or p53 ${ }^{\mathrm{R} 273 \mathrm{H}}$ were injected into the tail vein of NSG mice (Fig. 5a). Remarkably, 9 weeks after injection, the lungs of the mice injected with $\mathrm{p} 53^{\mathrm{R} 273 \mathrm{H}}$-overexpressing cells displayed a significantly larger area of lung metastases than in mice injected with $\mathrm{p} 53^{\mathrm{R} 175 \mathrm{H}}$ overexpressors (Fig. $5 \mathrm{~b}, \mathrm{c}$ ). Moreover, to better recapitulate CRC biology, we orthotopically injected SW480 cells harboring the two p53 mutants into the cecal wall of NSG mice (Fig 5d). Seven weeks later, mice were sacrificed and evaluated for distant organ metastases. Notably, four out of five mice in the R273H group developed both lung and liver metastases, 
while no metastases were observed in any of the mice injected with $\mathrm{p} 53^{\mathrm{R} 175 \mathrm{H}}$ overexpressors (Fig $5 \mathrm{e}, \mathrm{f}$ ). Thus, $\mathrm{p} 53^{\mathrm{R} 273 \mathrm{H}}$ not only confers increased migration and invasion in vitro, but also preferentially promotes metastatic behavior in vivo.

\section{$\mathrm{p} 53^{\mathrm{R} 273 \mathrm{H}}$ is recruited to $\mathrm{R} 273$ signature genes and activates them via its transactivation domain}

To explore the molecular mechanisms driving the transcriptional upregulation of R273 signature genes by $p 53^{\mathrm{R} 273 \mathrm{H}}$, we interrogated published p53 CHIP-seq data of SW480 cells (25), which express endogenous p53 ${ }^{\mathrm{R} 273 \mathrm{H}}$ (along with $\mathrm{p53}{ }^{\mathrm{P} 309 \mathrm{~S}}$ ). Remarkably, analysis of all mutp53 peaks using GREAT (35), revealed that the most significantly enriched cellular components associated with those peaks were related to cytoskeleton structure and function (Fig. 6a). Moreover, the mutp53 chromatin binding peaks were significantly correlated with the genes upregulated upon $\mathrm{p} 53^{\mathrm{R} 273 \mathrm{H}}$ overexpression in our SW480 RNA-seq (Fig. 6b), suggesting that regulation of their expression by $\mathrm{p} 53^{\mathrm{R} 273 \mathrm{H}}$ is mediated, at least in part, via the recruitment of $p 53^{\mathrm{R} 273 \mathrm{H}}$ to the corresponding chromatin regions. To query experimentally this notion, we compared by ChIP-qPCR the binding of $p 53^{\mathrm{R} 273 \mathrm{H}}$ and $p 53^{\mathrm{R} 175 \mathrm{H}}$ to regulatory elements of representative R273 signature genes, in SW480 cells ectopically expressing either mutant. As seen in Fig. $6 \mathrm{c}, \mathrm{p} 53^{\mathrm{R} 273 \mathrm{H}}$ indeed displayed significantly stronger binding than $\mathrm{p} 53^{\mathrm{R} 175 \mathrm{H}}$ to those regulatory regions.

Previous work has demonstrated that $\mathrm{p} 53^{\mathrm{R} 273 \mathrm{H}}$ can act as a potent transcriptional activator when recruited to DNA, e.g. as a GAL4 fusion protein $(36,37,38)$. The N-terminal transactivation domain (TAD) is essential for this activity (36). In agreement, while transiently-transfected $\mathrm{p} 53^{\mathrm{R} 273 \mathrm{H}}$ augmented the expression of endogenous R273 signature genes in p53KO SW480 cells, this effect was lost when the cells were transfected with a TAD-mutated version of $p 53^{\mathrm{R} 273 \mathrm{H}}$ (Fig. $6 \mathrm{~d}$ and Fig. S6a), despite being expressed at comparable amounts in the transfected cells (Fig. 6e,f). In addition to p53 ${ }^{\mathrm{R} 273 \mathrm{H}}$, the $p 53^{R 273 C}$ mutation is also fairly common in human cancer, including CRC. As seen in Fig. S6b,c, p53 ${ }^{R 273 C}$ was also capable of transactivating endogenous R273 signature genes in transiently transfected p53KO SW480 cells.

Collectively, these observations support the notion that recruitment of R273-mutated p53 proteins to specific chromatin regions alters the expression of associated genes, in a TAD-dependent manner. These transcriptional alterations may underpin the observed biological effects of the R273 mutants, leading to enhanced tumor progression and worse patient outcome.

\section{Disscusion}

The abundance of TP53 mutations and the increasing amount of clinical and genomic data derived from cancer patient tumors represent an opportunity to better understand the impact of different TP53 mutants on the features of the tumors that harbor them. Such understanding may potentially help in translating TP53 status information into better individualized treatment decisions. This is particularly 
relevant for CRC, where the frequency of TP53 missense mutations, and especially hotspot mutations, is very remarkable.

In the present study, we compared the effects in CRC of two prevalent TP53 mutations, representing distinct types of mutp53 proteins. We show that R273 mutations direct a unique transcriptional program, which is not expressed in p53-null CRC cells or in tumors harboring truncating TP53 mutations, and thus constitutes a GOF activity of R273 mutants. Importantly, this program, which entails activation of critical cancer-related pathways associated with cytoskeleton function, cell invasion and metastatic properties, is not shared with R175 mutants. This corresponds to clinical data from multiple CRC cohorts, suggesting that R273 mutants are associated with accelerated cancer progression and overall more aggressive disease. Mechanistically, this appears to entail differential recruitment of R273 mutants to specific regulatory elements on the DNA. Most probably, such recruitment is not direct, relying on the preferential association of R273-mutated p53 with sequence-specific DNA binding proteins $(7,39,40)$.

Although many of the published studies on mutp53 GOF have focused on common features shared by multiple mutants $(41,42,43,44,45,46,47)$, differential effects of different hotspot mutants have also been described $(11,48,49,50)$, including quantitative differences in their interaction with critical partner proteins $(51,52)$. Of note, a recent study employing HCT116 CRC cells showed that $\mathrm{p} 53^{\mathrm{R} 273 \mathrm{H}}$ is a more potent enhancer of cancer cell stemness than other $\mathrm{p} 53$ hotspot mutants, owing to selective regulation of a subset of long noncoding RNAs (53). We now show that the differences between mutants go beyond molecular features and may actually dictate different patient survival. Moreover, we show that selective mutp53 GOF effects can be abolished by a specific pathway inhibitor, suggesting that patients whose tumors harbor different p53 mutants might react differently to the same treatment protocol. Hence the particular TP53 mutation, not just the presence or absence of TP53 mutations, may be of future value when devising individualized treatment strategies for CRC, and most probably also for other cancer types.

Surprisingly, in our study $\mathrm{p} 53^{\mathrm{R} 175 \mathrm{H}}$ did not exert measurable effects on the transcriptional landscape and biological features of SW480 cells. This was unexpected, given that R175 mutations are very frequent in CRC: if they have no contribution to this type of cancer, why are they seen so often? A trivial explanation might be that they merely occur at high frequency because of particular mutation signatures inherent to CRC, without any acquired GOF (9). Yet, a more appealing possibility is offered by the fact that R175 mutations are strongly associated with the CMS2 transcriptional signature (Fig. S4c). CMS2 tumors are characterized by WNT and MYC signaling activation (26). If p53 R175 mutants facilitate such activation, they are expected to promote CRC initiation and rapid primary tumor growth. Indeed, R175 mutations are more prevalent than R273 mutations in early stages of the disease, but become less prevalent at late stages, when invasive and metastatic capacities take the lead role (Fig. 1b). Furthermore, CMS2 tumors tend to be more "immune cold", displaying minimal expression of immune-related transcripts and low infiltration of immune cells $(54,55)$. It is conceivable that this may be partly due to GOF effects of mutp53, as suggested recently for pancreatic cancer (44). In such scenario, one might propose that R175 mutants may be particularly potent facilitators of immune evasion at early stages of CRC development, favoring their high abundance at those stages. 
Still, it is surprising $p 53^{\mathrm{R} 175 \mathrm{H}}$ hardly affected at all the SW480 transcriptome, despite being abundantly expressed. The most plausible explanation is that the effects of distinct p53 mutants are highly contextdependent. SW480 cells carry endogenous $\mathrm{p} 53^{\mathrm{R} 273 \mathrm{H}}$, and their transcriptional profile is consistent with the R273 signature and hence with the CMS4 program. Presumably, their intrinsic signaling context has been evolutionarily optimized to support the transcriptional and biological GOF effects of their endogenous $\mathrm{p} 53^{\mathrm{R} 273 \mathrm{H}}$, while concomitantly becoming non-supportive of alternative programs driven by other mutants such as $\mathrm{p} 53^{\mathrm{R} 175 \mathrm{H}}$, which are characteristic of CMS2 tumors. This conjecture is in line with broader evidence for context-dependent GOF effects of missense mutp53 proteins. For example, whereas a particular subset of p53 mutants is selectively enriched in vivo, consistent with GOF, these mutants are not enriched and do not reveal any GOF properties when the same cells are grown in vitro $(56,57)$. A striking example of the context dependency of p53 mutations in CRC has recently been described by showing that the gut microbiome can dictate whether mutp53 proteins enhance tumor growth or, conversely, even restrict it, displaying surprising tumor suppressor features (58). Intriguingly, even the R273 mutant, which we show here to exert distinct GOF effects, did not exhibit measurable GOF effects in a genetically modified mouse model of CRC (59), further demonstrating that the contribution of a particular p53 mutation to cancer progression is highly context-dependent.

The role of adjuvant therapy for colon cancer patient with stage 2 tumors remains unclear. Today, decisions regarding adjuvant therapy include estimation of recurrence risk assasment through high-risk clinicopathologic features (60). Our data suggest that CRC pateints with the R273 mutation are prone to advance to late stage disease and therfore might benefit from adjuvant therapy in this stage. Given that TP53 mutations are the most frequent single gene mutations in human cancer and that practically all current analytical cancer gene panels include TP53, provide an opportunity for a better treatment decision making for stage 2 colorectal patients.

Altogether, our findings argue that different p53 mutants may impart non-identical features on tumors, eventually impacting patient management and treatment decisions. Better understanding of such differential contributions of distinct p53 mutants and their context dependency is bound to make information on TP53 mutations more valuable in the future and hold great potential for better precisionbased medicine in the future.

\section{Methods}

\section{Data Acquisition and Processing}

TP53 somatic mutation status and clinical attributes from the DFCI, CPTAC-2 and MSKCC cohorts (16, $17,19)$ were retrieved from the CBioPortal open Platform. TP53 somatic mutation status and clinical attributes from the TCGA and ICGC (CRC cohorts) were downloaded from UCSC Xena Browser http://xena.ucsc.edu/. TP53 somatic mutation status and clinical attributes from GECCO and CCFR were taken from published data (18). All patients were grouped according to their TP53 status. 
TCGA RNA-Seq expression profiles ((HT-Seq count, log2(fpkm-uq+1) for normalization)), were downloaded from UCSC Xena Browser http://xena.ucsc.edu/. TCGA colon adenocarcinoma (TCGACOAD) and rectal adenocarcinoma (TCGA-READ) samples were filtered for primary tumour samples and divided according to their TP53 status into R175 mutant tumours, R273 mutant tumours and Truncated tumours (comprised of tumours with frameshift, nonsense and splice site mutations).

RNA-seq data and gene somatic mutations data from cancer cell lines was downloaded from Xena Browser (CCLE dataset, RPKM), filtered for large intestine cell lines and divided into groups according to their TP53 status.

\section{Cell Lines, transfections and viral infections}

Cells were maintained at $37^{\circ} \mathrm{C}$ with $5 \% \mathrm{CO}_{2}$. SW480 and RKO cells were cultured in DMEM (Biological Industries, BI), COLO-205 cells were grown in RPMI (BI) and HCT116 cells were grown in McCoy's 5A (Sigma). All culture media were supplemented with $10 \% \mathrm{FBS}(\mathrm{BI})$ and $1 \%$ penicillin-streptomycin (BI). All cell lines were tested negative for Mycoplasma. SW480 TP53 knockout cells and RKO TP53 knockout cells were a kind gift from Varda Rotter (Weizmann Institute of Science). HCT116 TP53 knockout cells were a kind gift from Keren Vousden (Francis Crick Institute).

Plasmid transfection was done with the jetPEI DNA transfection reagent (Polyplus Transfection). The final DNA amount was $2 \mu \mathrm{g}$ per well in a 6-well plate, and the transfection medium was replaced after 24 hours. Cells were collected 48 hours after transfection for gene expression profiling by RT-qPCR. pCB6, pCB6-R273H and pCB6-R273H with substitutions of residues 22 and 23 (L22Q/W23S; R273H TAD mutant), were a generous gift from Keren Vousden.

For lentivirus infections, SW480, HCT116, and RKO TP53 knockout cells and CACO-205 parental cells were infected with recombinant lentiviruses (pEF1alpha-p53 R273H IRES-EGFP and pEF1alphap53R175H IRES-EGFP) to express the corresponding mutant proteins. Lentiviral packaging was performed by jetPEl-mediated transfection of Phoenix cells with the indicated plasmid DNAs, together with a plasmid encoding the VSVG envelope protein and packaging plasmids. Virus-containing supernatants were collected $48 \mathrm{~h}$ and $72 \mathrm{~h}$ after transfection, filtered, and supplemented with $8 \mu \mathrm{g} / \mathrm{ml}$ polybrene (Sigma). One week post infection, cells were subjected to FACS sorting for GFP positive cells. Alternatively, SW480 cells were infected with recombinant lentiviruses (pLK0.1-puro-shp53, TRCN0000010814 (Sigma) to produce shRNA directed against the 3' UTR of the endogenous mutant p53 mRNA, together with recombinant lentiviruses (pEF1alpha-p53 R273H IRES-EGFP and pEF1alphap53R175H IRES-EGFP) to express the corresponding mutant proteins. 48h after infection, p53 knockdown cells were selected with puromycin, and one week later were subjected to FACS sorting for GFP positive cells.

p53 knockdown and mutant protein expression were verified by RT-qPCR and Western blot analysis.

\section{Immunoblotting}


Cell pellets were resuspended in RIPA (Radioimmunoprecipitation assay) buffer, and protein sample buffer was added after centrifugation. Samples were boiled and resolved by SDS-PAGE. The following antibodies were used: GAPDH (Cell signaling, 14C10), p53 (mixture of monoclonal antibodies D01 + PAb1801). Imaging and quantification were performed using ChemiDoc MP Imager with Image Lab 4.1 software (Bio-Rad).

\section{Time-lapse microscopy.}

Cells were plated in 6 well plastic bottom dishes and monitored by time-lapse imaging using a Celldiscoverer 7 microscope (Carl Zeiss Ltd.) Imaging was performed using the oblique contrast method through a Plan- Apochromat 20X/0.7 and a 0.5x Tubelens (effective magnification of 5X and 0.35NA). Illumination was done with a white-light LED set to $10 \%$ and detection by a 14 bit Axiocam 506 CCD camera (Carl Zeiss Ltd.) with $10 \mathrm{~ms}$ exposure time. Pixel size was $0.462 \mathrm{~m} \times 0.462 \mathrm{~m}$. Image tiling was used in order to cover a large area. Images were taken at 1 hour intervals, for total of 24 hours.

To quantify the cell shape, we segmented the cells using the ilastik Boundary based segmentation with Multicut workflow (61). We trained in ilastik 1) auto-context pixel classifier for 3 classes:

boundary/cell/background and 2) multi-cut edge classifier. These were then applied sequentially to all the images in batch. We wrote a Fiji (62) macro to select cells from the multi-cut objects based on their size (between minimum and maximum values) and their average probability of belonging to the "cell" class of the ilastik auto-context pixel classifier. We discarded cells touching the border of the image. For each cell, we measured the aspect ratio (AR) - the ratio between the major and minor axis of the best-fitted ellipse. Spread cells were defined as those with $A R>1.8$. For each time point, the percentage of spread cells out of the total number of detected cells was calculated.

\section{RNA-seq}

SW480 TP53 knockout cells and SW480 cells stably expressing R175H and R273H mutant protein were seeded at a density of 1.5 million per 10 centimeter dish, and RNA was extracted either 6 hours or 24 hours post seeding, using a NucleoSpin kit (Macherey Nagel). RNA of SW480 cells with stable p53 knockdown or overexpression of shRNA -resistant $\mathrm{p} 53^{\mathrm{R} 175 \mathrm{H}}$ or $\mathrm{p} 53^{\mathrm{R} 273 \mathrm{H}}$ was extracted similarly.

RNA-seq libraries were prepared at the Crown Genomics Institute of the Nancy and Stephen Grand Israel National Center for Personalized Medicine, Weizmann Institute of Science. A bulk adaptation of the MARS-Seq protocol (24) was used to generate RNA-seq libraries for expression profiling. Briefly, $30 \mathrm{ng}$ of input RNA from each sample was barcoded during reverse transcription and pooled. Following Agencourct Ampure XP beads cleanup (Beckman Coulter), the pooled samples underwent second strand synthesis and were linearly amplified by T7 in vitro transcription. The resulting RNA was fragmented and converted into a sequencing-ready library by tagging the samples with Illumina sequences during ligation, RT and PCR. Libraries were quantified by Qubit and TapeStation as well as by qPCR for GAPDH as previously described (24). Sequencing was done with a Nextseq 75 cycles high output kit (Illumina). 
Heatmaps were generated with Partek Genomics Suite 7.0 (Partek Inc.), using log normalized values (rld), with row standardization and Euclidean clustering

\section{Gene Set Enrichment Analysis}

Gene Set Enrichment Analysis (GSEA) (63), was employed to determine whether the R273 gene signature exhibits a statistically significant bias in its distribution within a ranked gene list. We followed the standard procedure as described in the GSEA user guide

(http://www.broadinstitute.org/gsea/doc/GSEAUserGuideFrame.html) to create the ranked gene list for RNA-seq profiling of our data/published data/TCGA data, and tested the R273 signature for significant differences in distribution. The FDR for GSEA is the estimated probability that a gene set with a given NES (normalized enrichment score) represents a false-positive finding.

\section{RT-qPCR}

RNA was isolated using the NucleoSpin kit (Macherey Nagel). $1 \mu \mathrm{g}$ of each RNA sample was reverse transcribed using Luna ${ }^{\circledR}$ Universal qPCR Master Mix (New England Biolabs). Real-time qPCR was performed using SYBR Green PCR Supermix (Invitrogen) with a StepOne real-time PCR instrument (Applied Biosystems). For each gene, values for the standard curve were measured and the relative quantity was normalized to GAPDH mRNA. Primers are listed in Supplementary Table 4.

\section{RhoGTPase activity assay.}

Endogenous activity of RhoA, Rac1 and Cdc42 levels was determined by using an enzyme-linked immunosorbent assay (ELISA)-based G-LISA kit (Cytoskeleton, Inc \#BK135) strictly following the manufacturer's instructions. Briefly, Briefly, SW480 cells stably expressing $\mathrm{p} 53^{\mathrm{R} 175 \mathrm{H}}$ or $\mathrm{p} 53^{\mathrm{R} 273 \mathrm{H}}$ were plated and allowed to grow to $\sim 70 \%$ confluence before being washed with PBS and lysed in $100 \mu$ l of icecold lysis buffer in the presence of protease and phosphatase inhibitors. The lysate was clarified by centrifugation at $10,000 \times \mathrm{g}$ for $1 \mathrm{~min}$, and snap-frozen in liquid nitrogen. After normalizing protein concentration using PrecisionRed (Cytoskeleton, Inc), samples were added in triplicate to wells coated with a respective GTP-binding protein. After washing, bound GTPases levels were determined by subsequent incubations with a respective antibody and a secondary HRP-conjugated antibody, followed by addition to an HRP detection reagent. Background was determined by a negative control well. Absorbance was measured at a wavelength of $490 \mathrm{~nm}$ using a microplate reader (Thermo Fisher Scientific). Values are expressed as mean \pm SEM of Three technical replicates.

\section{Migration assays}

Migration assays were performed using the transwell system (8 $\mu \mathrm{m}$ pore size; Costar). In brief, 60,000 cells in either serum-free medium (RKO) or medium containing 1\% FBS (SW480) were seeded in the upper chamber, while the lower chamber was filled with 600 microliter of culture medium supplemented with $10 \%$ FBS. Cells were allowed to migrate for 24 hours (SW480) or 30 hours (RKO). Cells on the lower 
surface of the chamber were fixed with $4 \%$ PFA and stained with crystal violet. Cells on the upper surface were removed with cotton plugs. Stained cells were imaged with a Nikon Eclipse Ti-E microscope at $\times 4$ magnification, capturing at least three fields for each condition, and crystal violet stained areas were quantified with an ImageJ macro. Coverage by migrating cells was calculated as percentage of stained area relative to total area.

For MBQ-167 migration assay, SW480 cells were treated for 4 hours with either MBQ (750nM) or DMSO. After 4 hours, cells were trypsinized and placed in the upper transwell as above. 600 microliter of culture medium containing 10\% FBS and either MBQ-167 (750nM) or DMSO were added to the bottom chamber. 24 hours post seeding, cells were fixed and stained. Stained area was quantified as above.

\section{Invasion assays}

For invasion assays, 200,000 cells were seeded in transwell chambers pre-coated with Matrigel (Corning). 600 microliter of culture medium containing $10 \%$ FBS and supplemented with EGF $(100 \mathrm{ng} / \mathrm{ml})$ were added to the bottom chamber. After 24 hours cells were fixed and stained. Stained area was quantified as above.

\section{$\underline{\text { In vivo experiments }}$}

All animal experiments and methods were approved by the Weizmann Institutional Animal Care and Use Committee. For tail vein injection, $2.5^{\wedge} 10^{6}$ cells were resuspended in 100 microliter PBS before being injected through the tail vein. Tumours were harvested 9 weeks post-injection, as indicated in the corresponding figure legends. For orthotopic injection, $1^{\wedge} 10^{7}$ cells were resuspended in 50 microliter PBS, diluted in Matrigel (1:1), and injected into the cecal wall. Tumours were harvested 7 weeks post-injection.

\section{Chromatin Immunoprecipitation (ChIP) analysis}

Chromatin immunoprecipitation was performed as previously described (39). SW480-p53 ${ }^{\mathrm{R} 175 \mathrm{H}}$ and SW480-p53 ${ }^{\mathrm{R} 273 \mathrm{H}}$ cells at $70 \%$ confluence were subjected to crosslinking by adding $1 / 10$ volume of fresh $11 \%$ formaldehyde solution (50 mM HEPES-KOH pH7.5, 100 mM NaCl, 1 mM EDTA, 0.5 mM EGTA, 11\% formaldehyde) for $10 \mathrm{~min}$, followed by incubation in $0.125 \mathrm{M}$ glycine for $5 \mathrm{~min}$. DNA was sheared to a range of 100-600 bp by subjecting the chromatin to sonication in a Bioruptor sonicator (Diagenode). 1/10 of the chromatin sample was set aside as input. Mouse anti-p53 (Santa Cruz, D01, sc-126) and normal mouse IgG (Santa Cruz, sc-2025) were used for immunoprecipitation. Immune complexes were collected using Dynabeads protein $\mathrm{G}$ (Thermo Fisher Scientific). After reverse crosslinking and Proteinase $\mathrm{K}$ digestion, DNA was recovered using ChIP DNA Clean \& Concentrator columns (Zymo Research). qPCR was performed using Luna ${ }^{\circledR}$ Universal qPCR Master Mix (New England Biolabs) on a 7500 Fast RealTime PCR System (Thermo Fisher Scientific). Data was normalized by the $\Delta \Delta \mathrm{Ct}$ method over Input (1:20 dilution) and IgG samples. Sequences of the primers used for ChIP analysis are listed in Supplementary Table 4. 
For Genomic Regions Enrichment of Annotations Tool (GREAT), we used published ChIP-seq data (GEO Series Accession Number GSE102796). 17,980 peaks identified in two replicates were analyzed for GO cellular component enrichment using GREAT (35).

\section{Cell cycle profiling}

Cells were grown in $6 \mathrm{~cm}$ dishes for 24 hours, trypsinized, and subjected to cell cycle analysis with a Phase-Flow BrdU Cell Proliferation Kit (BioLegend). Briefly, cells were incubated with BrdU for 75 minutes and labeled with Alexa Fluor-647-conjugated anti-BrdU antibody. Total DNA was stained with DAPI. Then, 50,000 cells were collected and analyzed by multispectral imaging flow cytometry. The percentage of cells in each cell cycle phase was manually determined on the basis of BrdU intensity and total DNA content, using FlowJo (Becton, Dickinson and Company).

\section{Statistical data analysis}

Independent biological replicates were performed and group comparisons were done as detailed in the figure legends. $P$-values below 0.05 were considered significant. Statistical analysis was performed using the Graph-Pad Prism 9.1.0 software. Statistical significance between two experimental groups is indicated by asterisks; *, P $<0.05 ; * \star, P<0.01 ; * \star \star, P<0.001 ; * \star \star \star, P<0.0001$.

\section{Declarations}

\section{Acknowledgments}

We thank Benjamin Geiger for inspiring scientific discussions. We thank Carine Joubran for experimental help and Ron Rotkopf for statistical help. RNA-seq analysis was done with critical advice from Michal Pearl and Hadas Keren-Shaul of the Crown Genomics Institute of the Nancy and Stephen Grand Israel National Center for Personalized Medicine, Weizmann Institute of science. This work was supported in part by the Dr. Miriam and Sheldon G. Adelson Medical Research Foundation, a Center of Excellence grant No. 3165/20 from the Israel Science Foundation, the Robert Bosch Stiftung and the Berthold Leibinger Stiftung, the Thompson Family Foundation, and a grant from Anat and Amnon Shashua, and the Moross Integrated Cancer Center. M.O. is incumbent of the Andre Lwoff chair in molecular biology.

Author Contributions; O.H. and M.O. designed research; O.H. performed research; N.B., M.S., G.F., S.M., M.M., G.M, A.G and I.G helped with the experiments; E.F. and O.G. helped with the analyses; R.Y, Y.A, G.B., D.K., Y.Y. and M.O. supervised research; O.H., Y.A. and M.O. wrote the paper. All authors discussed the results and commented on the manuscript.

\section{Competing Interests statement}

The authors declare that they have no competing interests

\section{References}


1. Olivier, M., Hollstein, M. \& Hainaut, P. TP53 mutations in human cancers: origins, consequences, and clinical use. Cold Spring Harbor perspectives in biology 2, (2010).

2. Kandoth, C. et al. Mutational landscape and significance across 12 major cancer types. Nature 502, 333-339 (2013).

3. Donehower, L. A. et al. Integrated Analysis of TP53 Gene and Pathway Alterations in The Cancer Genome Atlas. Cell Rep. 28, 1370-1384.e5 (2019).

4. Oren, M. \& Rotter, V. Mutant p53 gain-of-function in cancer. Cold Spring Harbor perspectives in biology 2, (2010).

5. Freed-Pastor, W. A. \& Prives, C. Mutant p53: One name, many proteins. Genes Dev. 26, 1268-1286 (2012).

6. Muller, P. A. J. \& Vousden, K. H. Mutant p53 in cancer: New functions and therapeutic opportunities. Cancer Cell 25, 304-317 (2014).

7. Kim, M. P. \& Lozano, G. Mutant p53 partners in crime. Cell Death Differ. 25, 161-168 (2018).

8. Mantovani, F., Collavin, L. \& Del Sal, G. Mutant p53 as a guardian of the cancer cell. Cell Death and Differentiation 26, 199-212 (2019).

9. Baugh, E. H., Ke, H., Levine, A. J., Bonneau, R. A. \& Chan, C. S. Why are there hotspot mutations in the TP53 gene in human cancers? Cell Death and Differentiation 25, 154-160 (2018).

10. May, P. \& May, E. Twenty years of p53 research: Structural and functional aspects of the p53 protein. Oncogene 18, $7621-7636$ (1999).

11. Halevy, O., Michalovitz, D. \& Oren, M. Different tumor-derived p53 mutants exhibit distinct biological activities. Science (80-.). 250, 113-116 (1990).

12. Goldstein, I. et al. Understanding wild-type and mutant p53 activities in human cancer: New landmarks on the way to targeted therapies. Cancer Gene Therapy 18, 2-11 (2011).

13. Olive, K. P. et al. Mutant p53 gain of function in two mouse models of Li-Fraumeni syndrome. Cell $119,847-860$ (2004).

14. Rawla, P., Sunkara, T. \& Barsouk, A. Epidemiology of colorectal cancer: Incidence, mortality, survival, and risk factors. Przeglad Gastroenterologiczny 14, 89-103 (2019).

15. Kinzler, K. W. \& Vogelstein, B. Lessons from hereditary colorectal cancer. Cel/ 87, 159-170 (1996).

16. Giannakis, M. et al. Genomic Correlates of Immune-Cell Infiltrates in Colorectal Carcinoma. Cell Rep. 15, 857-865 (2016).

17. Vasaikar, S. et al. Proteogenomic Analysis of Human Colon Cancer Reveals New Therapeutic Opportunities. Cell 177, 1035-1049.e19 (2019).

18. Zaidi, S. H. et al. Landscape of somatic single nucleotide variants and indels in colorectal cancer and impact on survival. Nat. Commun. 11, 1-12 (2020).

19. Yaeger, R. et al. Clinical Sequencing Defines the Genomic Landscape of Metastatic Colorectal Cancer. Cancer Cell 33, 125-136.e3 (2018). 
20. Franko, J. et al. Treatment of colorectal peritoneal carcinomatosis with systemic chemotherapy: A pooled analysis of North Central Cancer Treatment Group phase III trials N9741 and N9841. J. Clin. Oncol. 30, 263-267 (2012).

21. Aoyagi, T., Terracina, K. P., Raza, A. \& Takabe, K. Current treatment options for colon cancer peritoneal carcinomatosis. World Journal of Gastroenterology 20, 12493-12500 (2014).

22. Damiens, K. et al. Clinical features and course of brain metastases in colorectal cancer: An experience from a single institution. Current Oncology 19, 254-258 (2012).

23. Berg, K. C. G. et al. Multi-omics of 34 colorectal cancer cell lines - a resource for biomedical studies. Mol. Cancer 16, 1-16 (2017).

24. Keren-Shaul, H. et al. MARS-seq2.0: an experimental and analytical pipeline for indexed sorting combined with single-cell RNA sequencing. Nat. Protoc. 14, 1841-1862 (2019).

25. Rahnamoun, H. et al. Mutant p53 shapes the enhancer landscape of cancer cells in response to chronic immune signaling. Nat. Commun. 8, (2017).

26. Guinney, J. et al. The consensus molecular subtypes of colorectal cancer. Nat. Med. 21, 1350-1356 (2015).

27. Sveen, A. et al. Colorectal cancer consensus molecular subtypes translated to preclinical models uncover potentially targetable cancer cell dependencies. Clin. Cancer Res. 24, 794-806 (2018).

28. Zhou, Y. et al. Metascape provides a biologist-oriented resource for the analysis of systems-level datasets. Nat. Commun. 10, 1-10 (2019).

29. Najm, P. \& El-Sibai, M. Palladin regulation of the actin structures needed for cancer invasion. Cell Adhesion and Migration 8, 29-35 (2014).

30. Yilmaz, M. \& Christofori, G. EMT, the cytoskeleton, and cancer cell invasion. Cancer and Metastasis Reviews 28, 15-33 (2009).

31. SUN, B., FANG, Y., LI, Z., CHEN, Z. \& XIANG, J. Role of cellular cytoskeleton in epithelial-mesenchymal transition process during cancer progression. Biomed. Reports 3, 603-610 (2015).

32. Parsons, J. T., Horwitz, A. R. \& Schwartz, M. A. Cell adhesion: Integrating cytoskeletal dynamics and cellular tension. Nature Reviews Molecular Cell Biology 11, 633-643 (2010).

33. Leve, F. \& Morgado-Díaz, J. A. Rho GTPase signaling in the development of colorectal cancer. Journal of Cellular Biochemistry 113, 2549-2559 (2012).

34. Haga, R. B. \& Ridley, A. J. Rho GTPases: Regulation and roles in cancer cell biology. Small GTPases 7, 207-221 (2016).

35. McLean, C. Y. et al. GREAT improves functional interpretation of cis-regulatory regions. Nat. Biotechnol. 28, 495-501 (2010).

36. Raj, N. \& Attardi, L. D. The transactivation domains of the p53 protein. Cold Spring Harb. Perspect. Med. 7, (2017).

37. Unger, T., Nau, M. M., Segal, S. \& Minna, J. D. P53: A transdominant regulator of transcription whose function is ablated by mutations occurring in human cancer. EMBO J. 11, 1383-1390 (1992). 
38. Raycroft, L., Schmidt, J. R., Yoas, K., Hao, M. M. \& Lozano, G. Analysis of p53 mutants for transcriptional activity. Mol. Cell. Biol. 11, 6067-6074 (1991).

39. Di Agostino, S. et al. Gain of function of mutant p53: The mutant p53/NF-Y protein complex reveals an aberrant transcriptional mechanism of cell cycle regulation. Cancer Cell 10, 191-202 (2006).

40. Strano, S. et al. Mutant p53: An oncogenic transcription factor. Oncogene 26, 2212-2219 (2007).

41. Capaci, V. et al. Mutant p53 induces Golgi tubulo-vesiculation driving a prometastatic secretome. Nat. Commun. 11, 1-19 (2020).

42. Sicari, D. et al. Mutant p53 improves cancer cells' resistance to endoplasmic reticulum stress by sustaining activation of the UPR regulator ATF6. Oncogene 38, 6184-6195 (2019).

43. Walerych, D. et al. Proteasome machinery is instrumental in a common gain-of-function program of the p53 missense mutants in cancer. Nat. Cell Biol. 18, 897-909 (2016).

44. Ghosh, M. et al. Mutant p53 suppresses innate immune signaling to promote tumorigenesis. Cancer Cell 39, 494-508.e5 (2021).

45. Solomon, H. et al. Mutant p53 gain of function underlies high expression levels of colorectal cancer stem cells markers. Oncogene 37, 1669-1684 (2018).

46. Liu, J., Zhang, C., Hu, W. \& Feng, Z. Tumor suppressor p53 and its mutants in cancer metabolism. Cancer Letters 356, 197-203 (2015).

47. Iwanicki, M. P. et al. Mutant p53 regulates ovarian cancer transformed phenotypes through autocrine matrix deposition. JCI Insight 1, 86829 (2019).

48. Solomon, H. et al. Various p53 mutant proteins differently regulate the ras circuit to induce a cancerrelated gene signature. J. Cell Sci. 125, 3144-3152 (2012).

49. Zhang, Y. et al. Somatic Trp53 mutations differentially drive breast cancer and evolution of metastases. Nat. Commun. 9, 3953-3953 (2018).

50. Humpton, T. J., Hock, A. K., Maddocks, O. D. K. \& Vousden, K. H. p53-mediated adaptation to serine starvation is retained by a common tumour-derived mutant. Cancer Metab. 6, 18 (2018).

51. Strano, S. et al. Physical interaction with human tumor-derived p53 mutants inhibits p63 activities. J. Biol. Chem. 277, 18817-18826 (2002).

52. Marin, M. C. et al. A common polymorphism acts as an intragenic modifier of mutant p53 behaviour. Nat. Genet. 25, 47-54 (2000).

53. Zhao, Y. et al. P53-R273H mutation enhances colorectal cancer stemness through regulating specific IncRNAs. J. Exp. Clin. Cancer Res. 38, 1-16 (2019).

54. Picard, E., Verschoor, C. P., Ma, G. W. \& Pawelec, G. Relationships Between Immune Landscapes, Genetic Subtypes and Responses to Immunotherapy in Colorectal Cancer. Frontiers in Immunology 11, (2020).

55. Roelands, J. et al. Immunogenomic classification of colorectal cancer and therapeutic implications. International Journal of Molecular Sciences 18, (2017). 
56. Kotler, E. et al. A Systematic p53 Mutation Library Links Differential Functional Impact to Cancer Mutation Pattern and Evolutionary Conservation. Mol. Cell 71, 178-190.e8 (2018).

57. Boettcher, S. et al. A dominant-negative effect drives selection of TP53 missense mutations in myeloid malignancies. Science (80-.). 365, 599-604 (2019).

58. Kadosh, E. et al. The gut microbiome switches mutant p53 from tumour-suppressive to oncogenic. Nature 586, 133-138 (2020).

59. Tang, J. et al. Trp53 null and R270H mutant alleles have comparable effects in regulating invasion, metastasis, and gene expression in mouse colon tumorigenesis. Lab. Investig. 99, 1454-1469 (2019).

60. Varghese, A. Chemotherapy for Stage II Colon Cancer. Clin. Colon Rectal Surg. 28, 256-261 (2015).

61. Berg, S. et al. ilastik: interactive machine learning for (bio)image analysis. Nat. Methods 16, 12261232 (2019).

62. Schindelin, J. et al. Fiji: An open-source platform for biological-image analysis. Nature Methods $\mathbf{9}$, 676-682 (2012).

63. Subramanian, A. et al. Gene set enrichment analysis: A knowledge-based approach for interpreting genome-wide expression profiles. Proc. Natl. Acad. Sci. U. S. A. 102, 15545-15550 (2005).

\section{Figures}


a

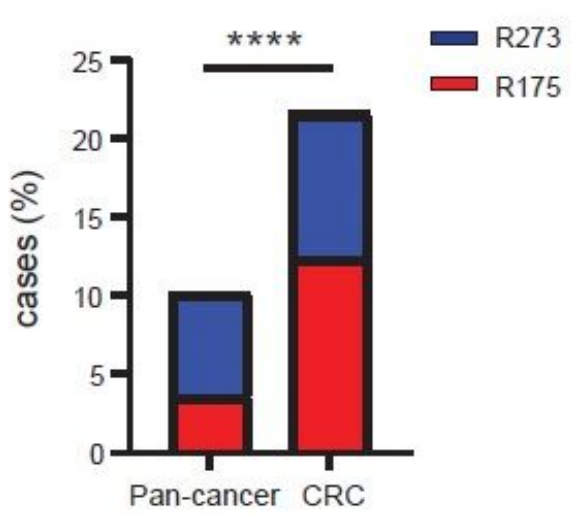

b

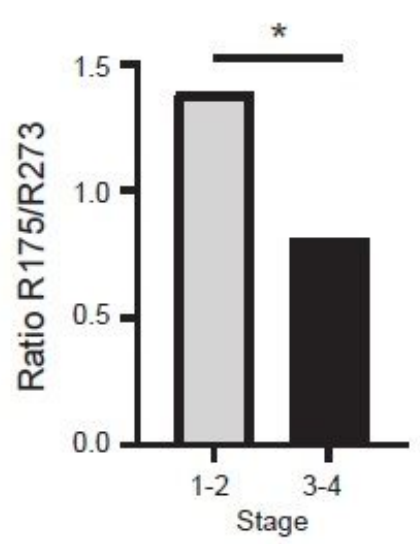

C

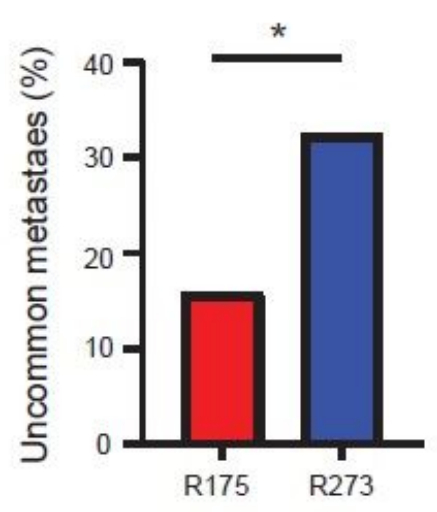

d

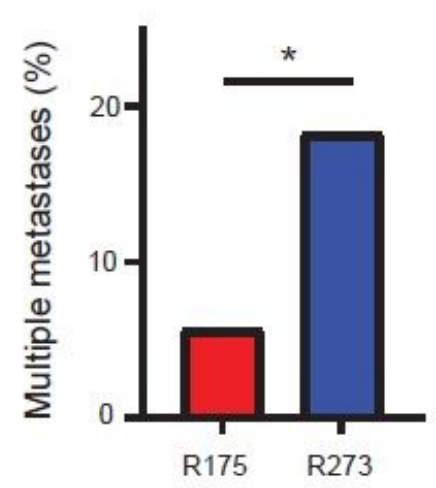

e

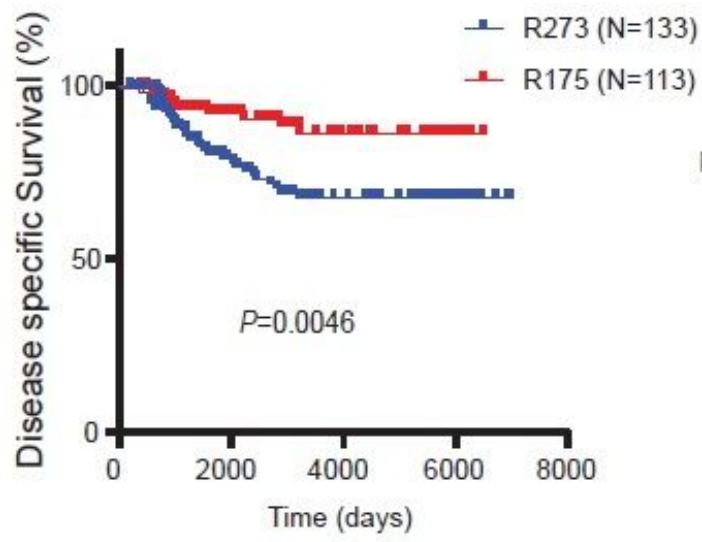

f

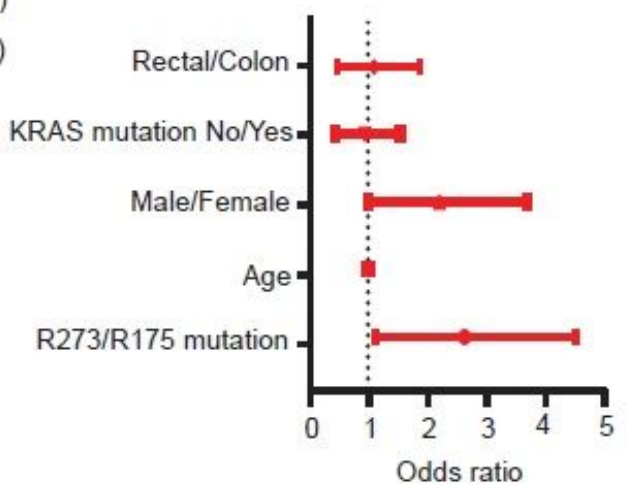

\section{Figure 1}

TP53 R273 mutations in CRC are preferentially associated with more aggressive cancer features and shorter overall survival a, Relative abundance of R175 and R273 TP53 hotspot mutations in colorectal cancer (CRC, $n=323$ ) versus all other cancers (Pan-cancer, $n=3396)$ in TCGA. Shown is the \% of cases with each hotspot mutation out of all TP53-mutated cases. $* \star \star \star P-v a l u e ~<0.0001$ (Fisher's exact test). b, Ratio between the numbers of CRC cases with R175 mutations and R273 mutations in stage 1-2 and in stage 3-4 disease. *P-value $<0.05$ (Fisher's exact test). c, Percentage of cases of each mutation type with metastases at uncommon sites (brain, bone, pelvis, peritoneum and omentum) at presentation, in the MSKCC cohort. *P-value $<0.05$ (Fisher's exact test). $d$, Percentage of cases of each mutation type with multiple metastases (three or more) at presentation, in the MSKCC cohort. *P-value $<0.05$ (Fisher's exact test). e, Disease specific overall survival of CRC patients with either R175 or R273 mutations. Compiled from TCGA COAD-READ and published data (17). Log-rank test. f, Multivariate cox regression analysis for the impact of multiple variables on overall survival in the patient collection described in (e). Circles represent hazard ratios and horizontal lines denote confidence intervals. 
a

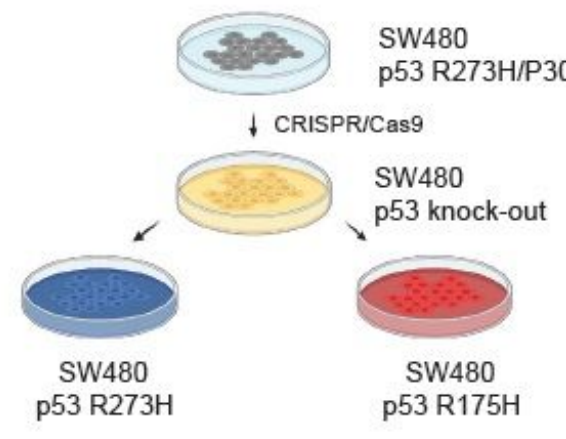

C

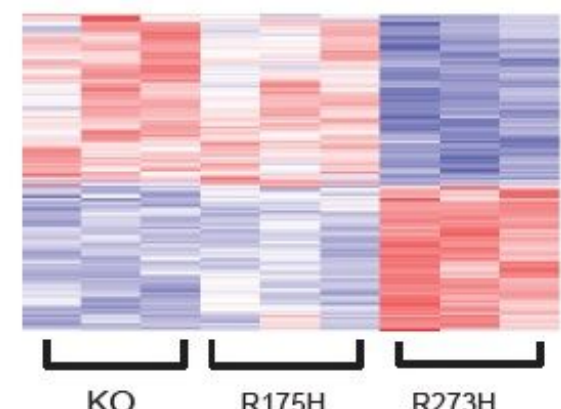

$\mathrm{KO}$
Color Key \begin{tabular}{lll}
\hline & 1 & 1 \\
\hline & & 3
\end{tabular} Row Z-score d Upregulated genes in $\mathrm{R} 273 \mathrm{H}$
compared to p53 knock-out

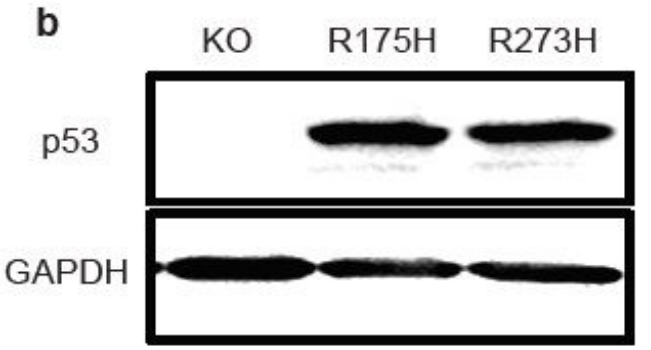

R273 signature

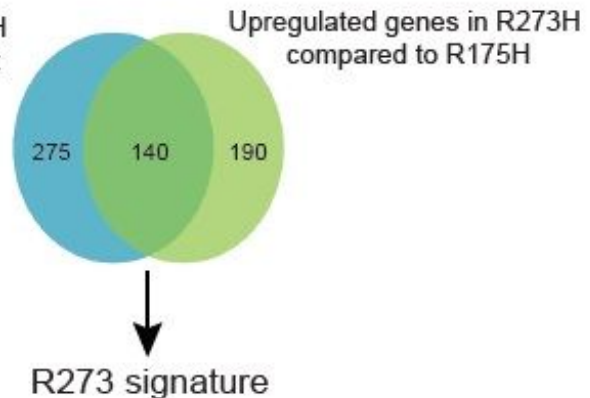

Upregulated genes in $\mathrm{R} 273 \mathrm{H}$ compared to $\mathrm{R} 175 \mathrm{H}$

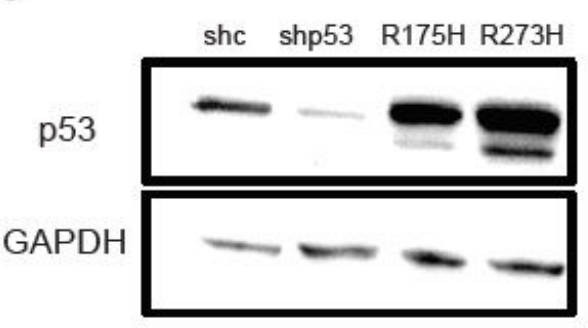

f

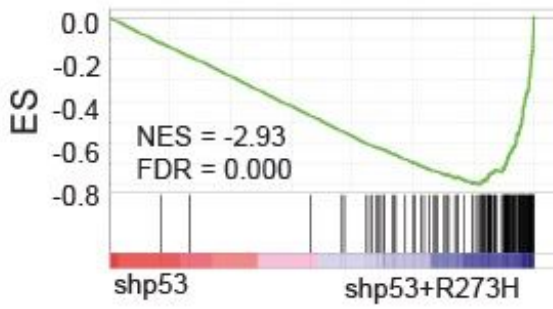

g

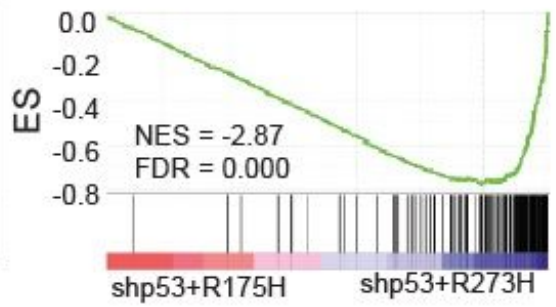

Figure 2

R273 mutants orchestrate a distinct transcriptional signature. a. SW480 cells in which the endogenous TP53 genes (harboring R273H and P309S mutations) had been knocked out, were stably transduced with p53R175H or p53R273H. b, Western blot analysis of p53 in SW480 knockout (KO) cells before and after transduction of p53R175H or p53R273H. c, SW480 TP53 KO cells and their derivatives expressing p53R175H or p53R273H were subjected to RNA-seq analysis. Shown is a heatmap of differentially expressed genes (fold change $>1.5$, pAdj $<0.05$ ) between p53 KO and p53R273H expressing cells. $d$, Venn diagram of upregulated genes (fold change $>1.5, \mathrm{pAdj}<0.1$ ) in p53R273H expressors relative to $\mathrm{p} 53 \mathrm{KO}$ cells (blue circle) or p53R175H expressors (green circle). The 140 overlapping genes were defined as the 'R273 signature'. e, Western blot analysis of p53 in SW480 cells stably transduced with shRNA directed against the 3' UTR of the TP53 gene (shp53), followed by stable overexpression of shRNA-resistant p53R $175 \mathrm{H}$ or $\mathrm{p} 53 \mathrm{R} 273 \mathrm{H}$. shc=SW480 cells transduced with control shRNA, to visualize the endogenous p53 protein. $f-g$, Gene Set Enrichment Analysis (GSEA) of differentially expressed genes in shp53 cells 
reconstituted with p53R273H vs control shp53 cells or shp53 cells reconstituted with p53R175H (ranked by fold change), using the R273signature as the tested gene set. ES=Enrichment score.
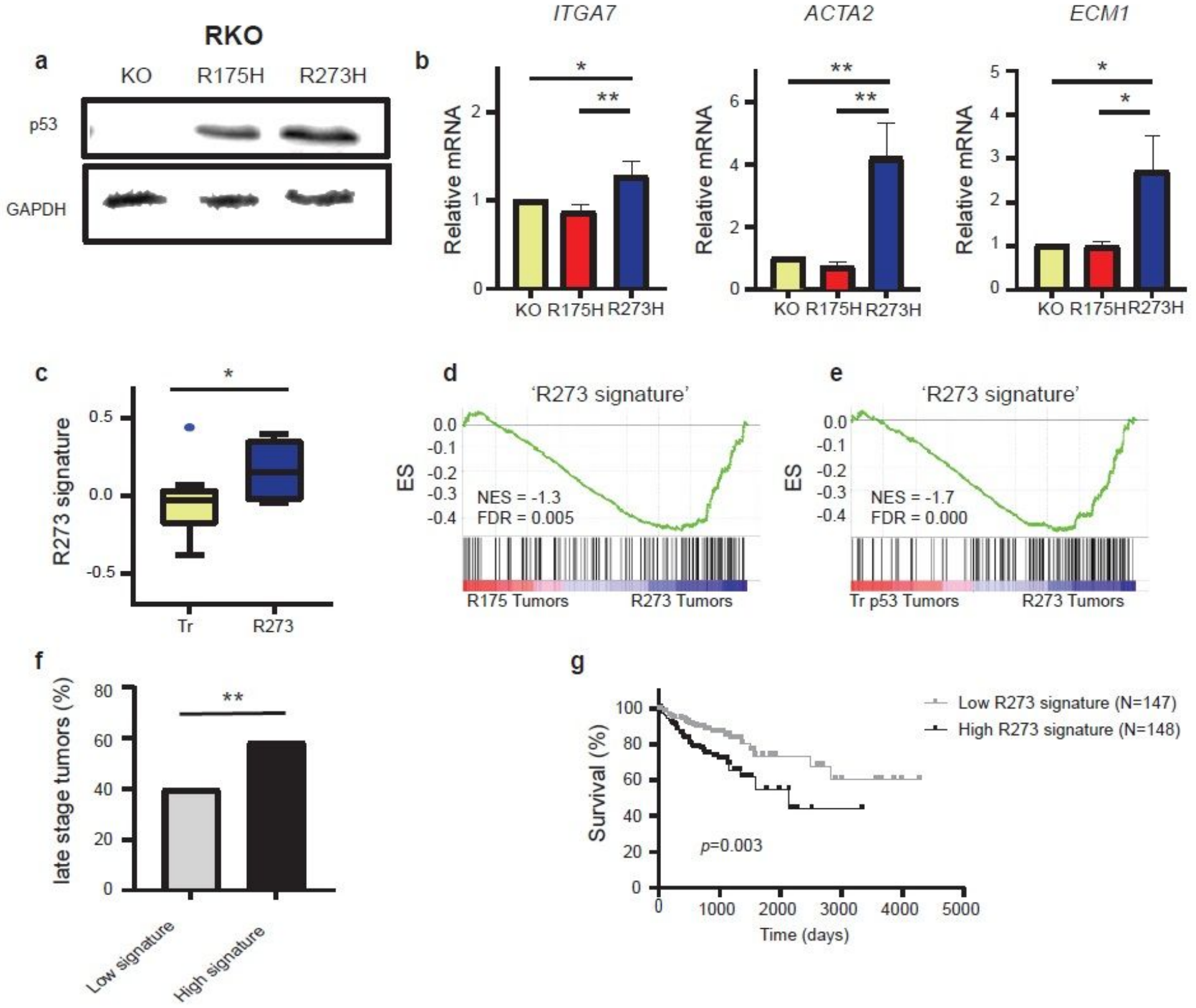

Figure 3

The R273 signature is upregulated in CRC cell lines and tumors and is associated with poor survival a, Western blot analysis of TP53 knockout (KO) RKO cells and their derivatives stably transduced with p53R175H or p53R273H. b, RT-qPCR analysis of the expression of representative R273 signature genes in the cells in (a). Values were normalized to GAPDH mRNA and are shown relative to the control KO cells. Mean + SEM from four independent repeats. *P-value $<0.05$; $* * P$-value $<0.01$ (one-way ANOVA and Tukey's post hoc test). c, Relative expression of the R273 signature in CRC cell lines harboring R273 mutations $(n=7)$ or truncating mutations $(T r ; n=11)$. Data accrued from Xena browser, Cancer Cell Line Encyclopedia (CCLE) RNA-seq gene expression data (RPKM). Before mean expression calculation, all genes in the R273 
signature were normalized to contribute equally to the signature. Fisher's exact test. d-e, GSEA of CRC tumors harboring R273 mutations ( $n=28)$ compared to tumors harboring R175 $(n=36)$ or truncating $(T r$; $\mathrm{n}=28$ ) mutations; for truncating mutations, we selected the 28 samples with the lowest p53 mRNA levels, to better approximate null mutations. Genes were ranked by fold change, and the R273 signature was used as the tested gene set. f. Percentage of late-stage (stage 3-4) tumors among CRC tumors in the lowest quartile $(n=173)$ or highest quartile $(n=174)$ of R273 signature expression. **P-value $<0.01$ (Fisher's exact test). g, Overall survival of patients within the highest or lowest quartile of R273 signature expression in the TCGA colorectal cancer cohort. Log-rank test.

a

regulation of plasma membrane bounded cell projection organization modulation of chemical synaptic transmission Rho protein signal transduction CXCR4 pathway regulation of synaptic plasticity cellular divalent inorganic cation homeostasis nucleosome positioning dilated cardiomyopathy leukocyte migration integrin activation CDC42 pathway

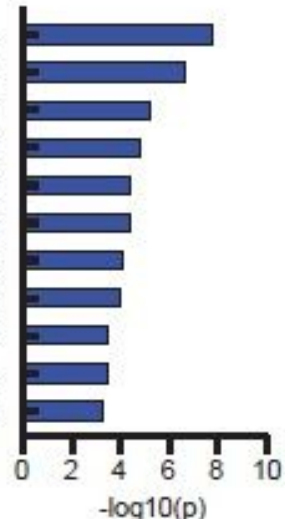

$-\log 10(p)$

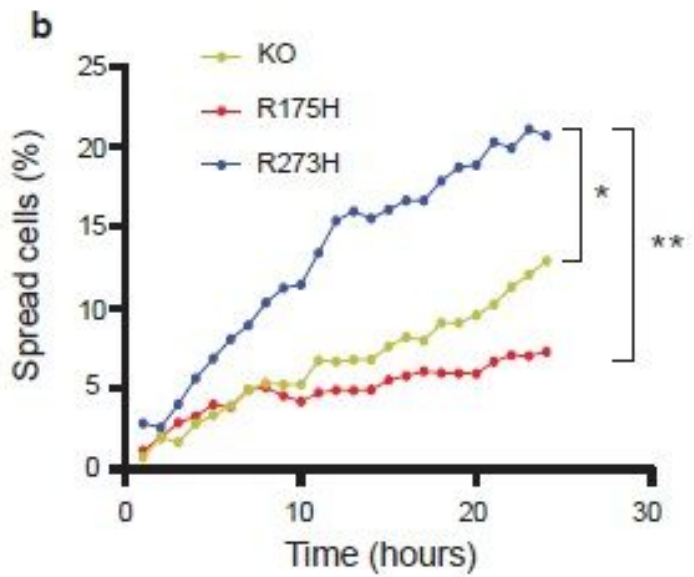

c

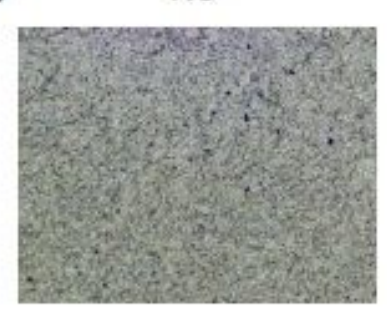

e

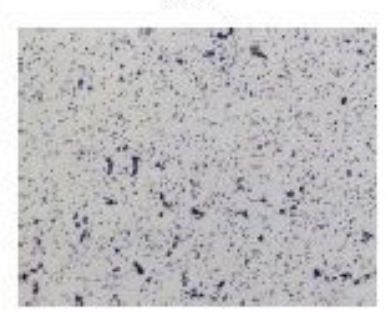

$\mathrm{R} 175 \mathrm{H}$

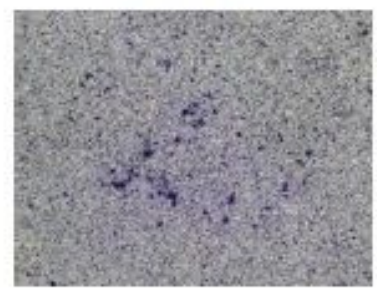

$\mathrm{R} 175 \mathrm{H}$

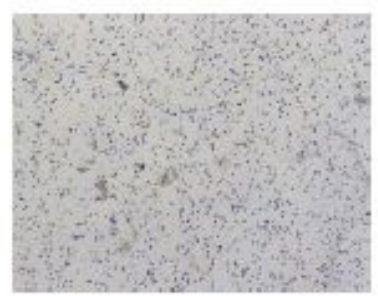

g

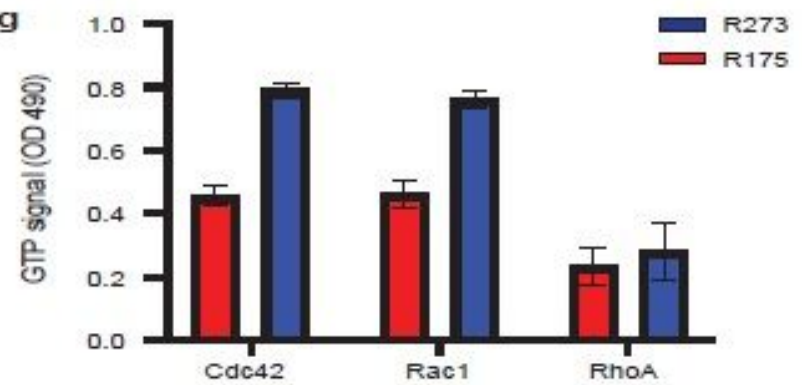

$\mathrm{R} 273 \mathrm{H}$

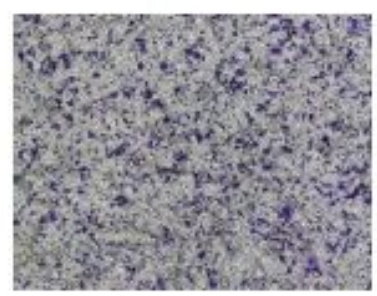

$\mathrm{R} 273 \mathrm{H}$

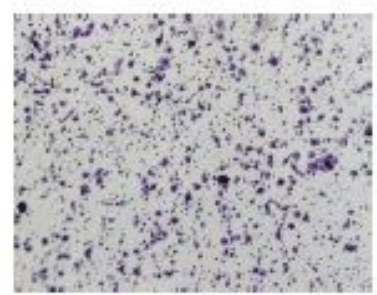

d
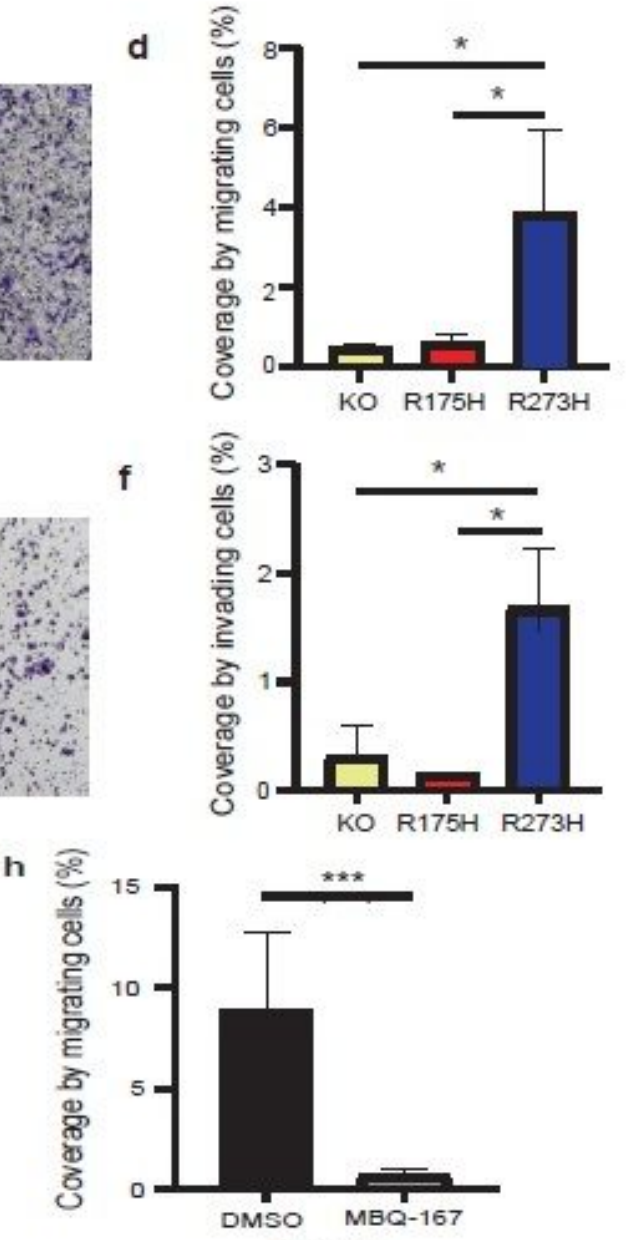

$\mathrm{R} 273 \mathrm{H}$ 


\section{Figure 4}

p53R273H promotes cell spreading, migration and invasion a, Gene Ontology analysis of the R273 signature (Metascape) b, Kinetics of spreading of SW480 p53 KO cells (KO) and their derivatives stably expressing p53R175H or p53R273H. Percentages of spread cells in the course of 24 hours were determined by time-lapse microscopy. Images were taken at 1 hour intervals, and were subjected to cell segmentation using the ilastik software. Aspect ratio was calculated for each segmented cells by ImageJ; a cell was considered spread if the aspect ratio was $>1.8$. Statistical analysis at $t=24$ was done using oneway ANOVA and Tukey's post hoc test. *P-value<0.05; $* *$ P-value $<0.01)$. c, Representative images of transwell migration assays performed with SW480 TP53 KO cells and their derivatives stably expressing p53R175H or p53R273H, 24 hours post-seeding. d, Average percentage of coverage (Image J) by migrating cells in transwell migration assays as described in (c). Three biological repeats. Nested ANOVA and Tukey's post hoc test of the indicated comparisons. e, Representative images of transwell invasion assays using Matrigel-coated inserts. $f$, Average percentage of coverage (ImageJ) by invading cells. Three biological repeats. Nested ANOVA and Tukey's post hoc test. g, SW480 cells stably expressing p53R175H or p53R273H were subjected to Rho signaling activation analysis using a G-LISA assay kit. Absorbance was read at $490 \mathrm{~nm}$. Three technical repeats. h, SW480 p53 KO cells stably expressing p53R273H were treated for 4 hours with either DMSO or MBQ-167 $(750 \mathrm{nM})$, and then subjected to a transwell migration assay as in (c), with or without MBQ-167. Average percentage of coverage by migrating cells (ImageJ) is shown. Four biological repeats. Nested ANOVA and Tukey's post hoc test. 
a
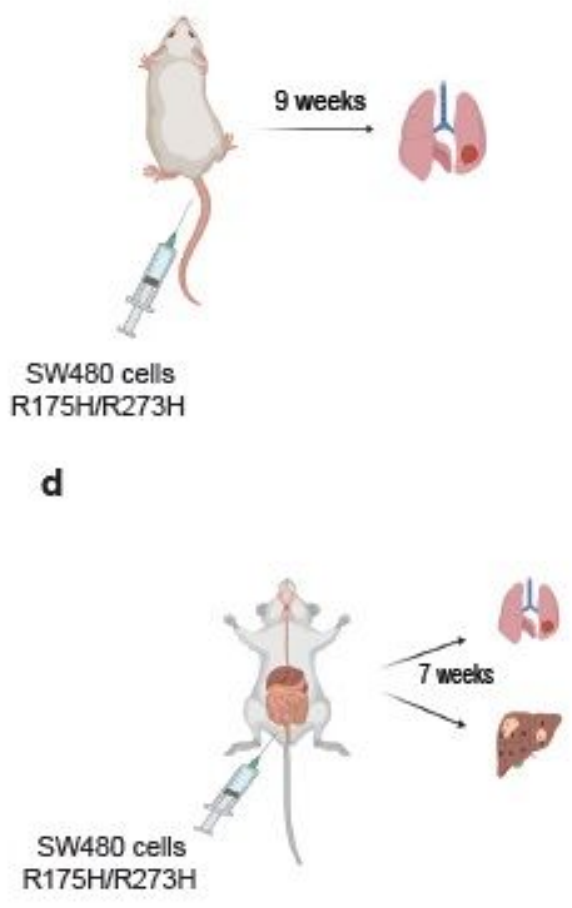

b

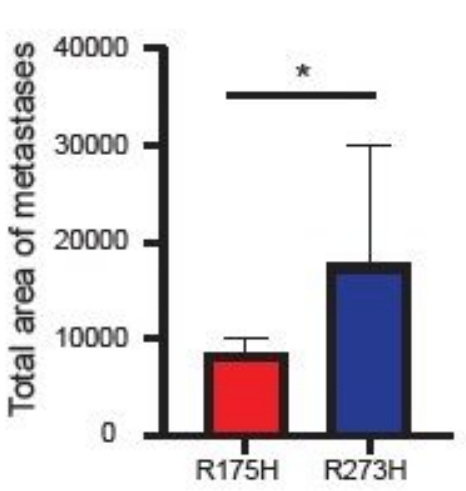

c

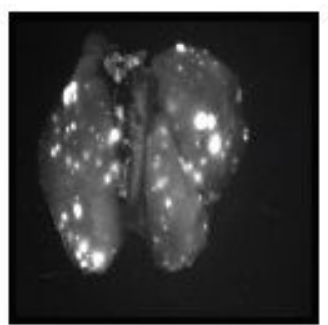

R175H

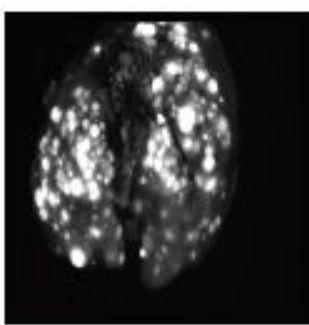

R273H

e

\begin{tabular}{|c|c|c|c|}
\hline & Liver & Lung & Peritoneum \\
\hline $\mathrm{R} 175 \mathrm{H}$ & $0 / 5$ & $0 / 5$ & $0 / 5$ \\
\hline $\mathrm{R} 273 \mathrm{H}$ & $4 / 5$ & $4 / 5$ & $1 / 5$ \\
\hline$P$-value & 0.0476 & 0.0476 & $\mathrm{~ns}$ \\
\hline
\end{tabular}

R175H

$\mathbf{f}$

UIVER
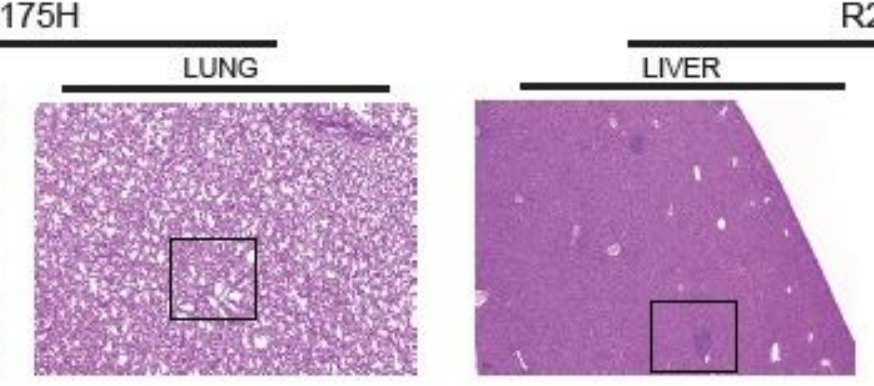

$\mathrm{R} 273 \mathrm{H}$
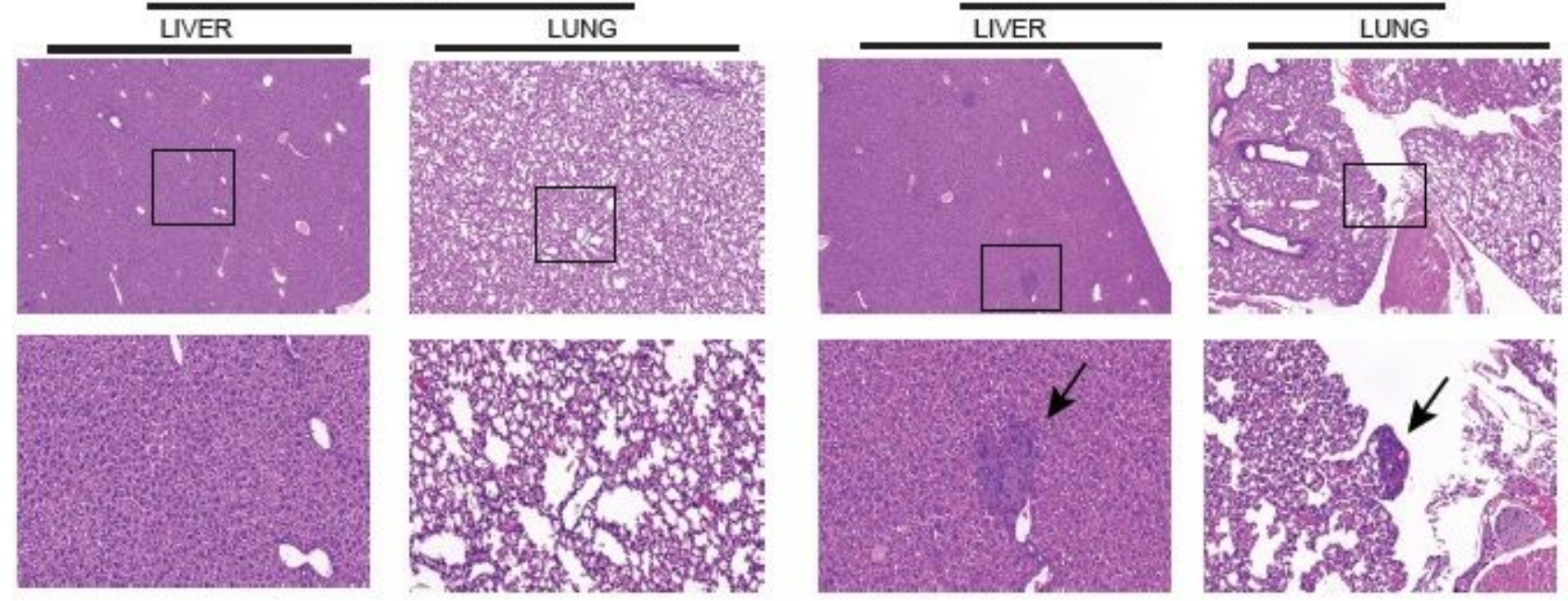

\section{Figure 5}

p53R273H preferentially promotes metastasis a, SW480 TP53 KO cells stably expressing p53R175H or p53R273H were injected into the tail vein of NSG mice. Lung metastases were evaluated at nine weeks post-injection. B. Total area of metastases at the lung surface (calibrated units), as quantified with ImageJ (n=5 mice per group). Two-tailed Mann-Whitney U-test. c, Representative images of lung metastases in mice analyzed as in (a). d, SW480 TP53 KO cells stably expressing p53R175H or p53R273H were injected into the cecal wall of NSG mice. Metastases were evaluated at 7 weeks postinjection. e, Numbers of mice with liver, lung, and peritoneal metastases in the groups described in (d). $f$, Representative H\&E staining images of lung and liver tissue of mice analyzed as in (d). The bottom row 
shows a 20X magnification of the areas marked by squares in the $5 \mathrm{X}$ magnification images in the upper row. Arrows indicate metastatic foci.

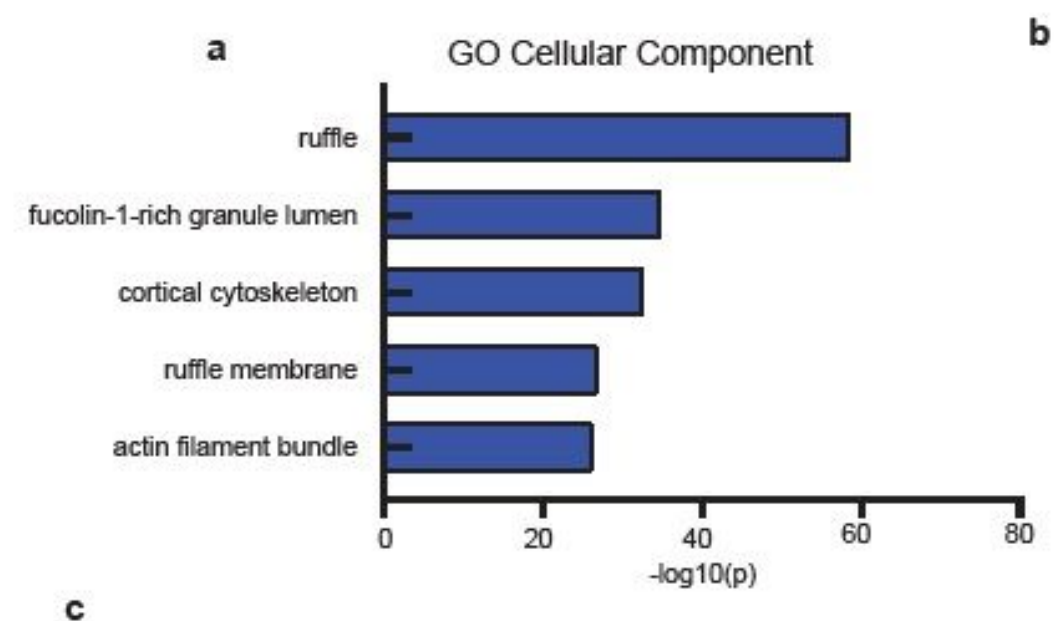

b
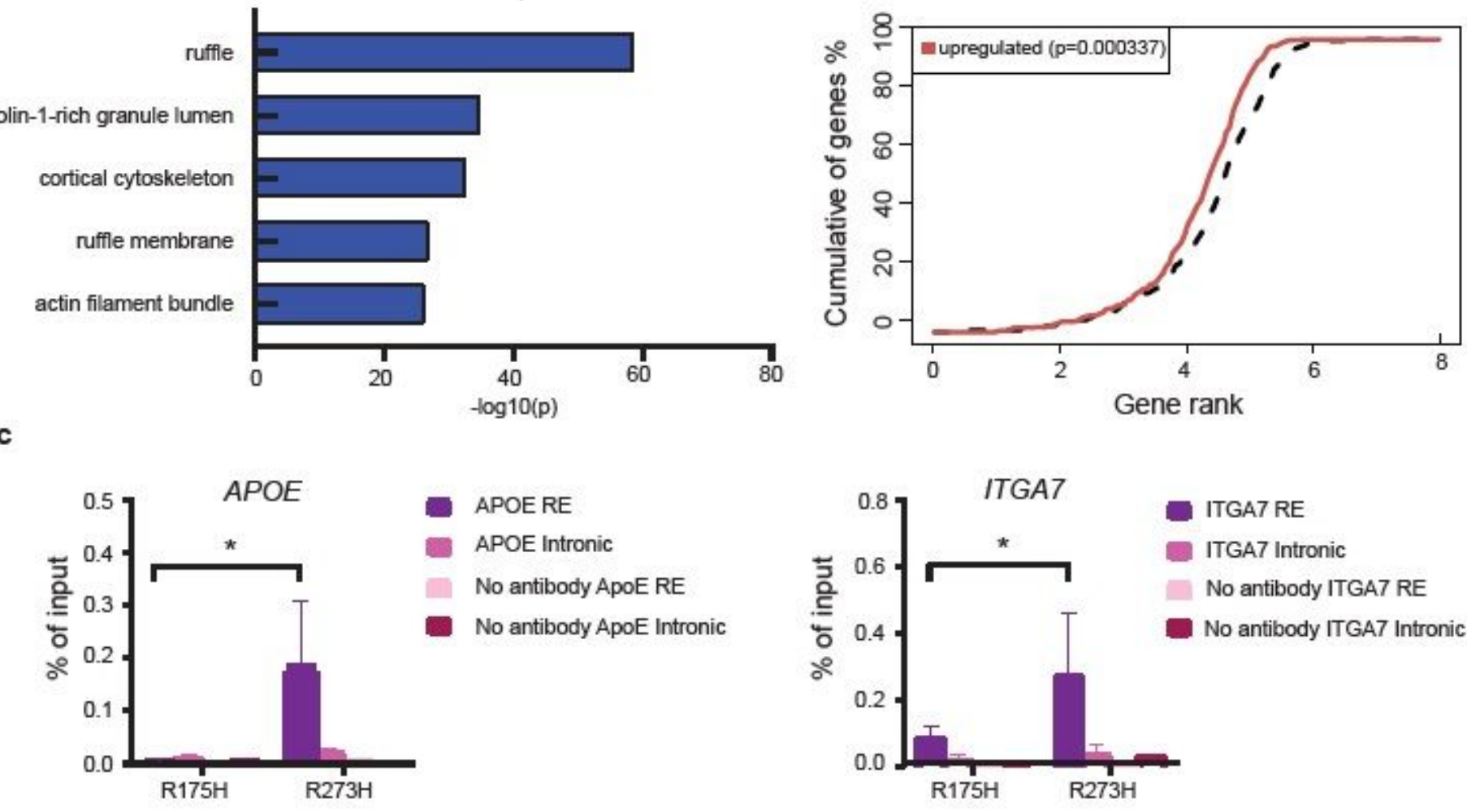

d

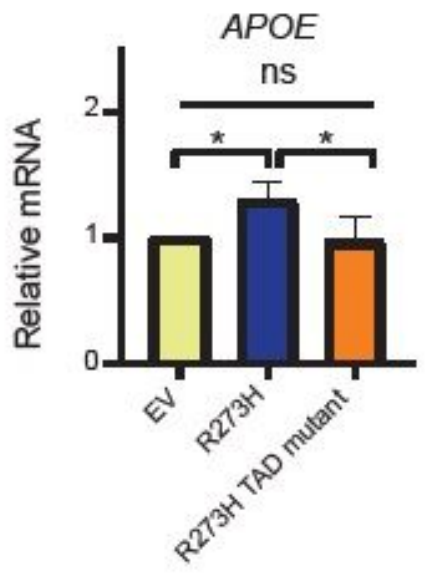

APOE RE

APOE Intronic

No antibody ApoE RE

No antibody ApoE Intronic e

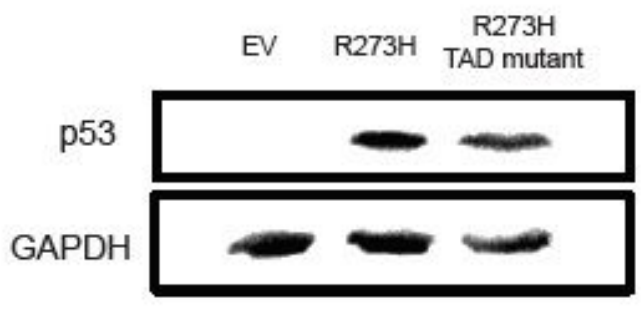

p53/GAPDH

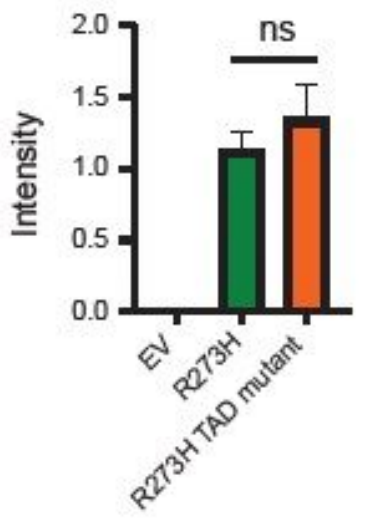

Figure 6

p53R273H binds gene regulatory elements and augments transcription a, Top five enriched GO cellular components associated with endogenous mutant p53 ChIP-seq peaks in SW480 cells. Data from Rahnamoun et al. (29) was subjected to analysis by GREAT as described in Methods. b, Mutant p53 chromatin binding peaks in SW480 cells are significantly associated with genes upregulated by p53R273H. All individual genes were ranked by their distance to the nearest p53 ChIP-seq peak in Rahnamoun et al. (29); the X-axis represents $\log (10)$ of the rank. Red line represents the genes upregulated in SW480 TP53 KO cells stably transduced with p53R273H, relative to control KO cells and 
cells transduced with $\mathrm{p} 53 \mathrm{R} 175 \mathrm{H}$ (fold change $>1.5$, pAdj $<0.1$; see Fig. $2 \mathrm{~d}$ ). Dashed line indicates all the other, non-differentially expressed genes as background. P-value indicates the significance of the difference between the upregulated genes and the non-differentially expressed genes (KolmogorovSmirnov test). c, ChIP-qPCR analysis of mutant p53 binding to regulatory regions of representative R273 signature genes in SW480 cells transfected with either $\mathrm{p} 53 \mathrm{R} 175 \mathrm{H}$ or p53R273H. Binding of mutant $\mathrm{p} 53$ to regulatory elements of ITGA7 and APOE is compared to binding to intronic regions of the same genes. Nested one way ANOVA and Tukey's post hoc test. d, RT-qPCR analysis of APOE mRNA in SW480 TP53 $\mathrm{KO}$ cells transiently transfected with empty vector control (EV), intact $\mathrm{p} 53 \mathrm{R} 273 \mathrm{H}$, or p53R273H harboring two mutations (L22Q and W23S) within the $\mathrm{p} 53$ transactivation domain (R273H TAD mutant). Cells were harvested 48 hours post-transfection. Values were normalized to GAPDH mRNA and are shown relative to the empty vector control cells. Mean + SEM from five independent biological repeats (one-way ANOVA and Tukey's post hoc test). e, Western blot analysis of p53 in SW480 KO cells transiently transfected with empty vector, intact p53R273H or p53R273H TAD mutant. f. Quantification of Western blot data as in (e), from two biological repeats, using Image Lab (Bio-Rad). One-way ANOVA and Tukey's post hoc test of the indicated comparisons.

\section{Supplementary Files}

This is a list of supplementary files associated with this preprint. Click to download.

- Supplementarymovielegends.docx

- Supplementarymovie1.mp4

- Supplementarymovie2.mp4

- Supplementarymovie3.mp4

- Tables14.docx

- SupplementaryFigures.pdf 